\title{
1 The human thalamus orchestrates neocortical oscillations during 2 NREM sleep.
}

3

4 Thomas Schreiner ${ }^{1}$, Elisabeth Kaufmann², Soheyl Noachtar ${ }^{2}$, Jan-Hinnerk 5 Mehrkens $^{3}$ \& Tobias Staudigl ${ }^{1}$

6

71 Department of Psychology, Ludwig-Maximilians-Universität München, Munich,

8 Germany

92 Epilepsy Center, Department of Neurology, Ludwig-Maximilians-Universität München, 10 Munich, Germany

11

123 Department of Neurosurgery, Ludwig-Maximilians-Universität München, Munich, 13 Germany

14

15

16 Correspondence: Tobias Staudigl (tobias.staudigl@|mu.de)

17

18 


\section{ABSTRACT}

20 A hallmark of non-rapid eye movement (NREM) sleep is the coordinated interplay

21 of slow oscillations (SOs) and sleep spindles. Traditionally, a cortico-thalamo-

22 cortical loop is suggested to coordinate these rhythms: neocortically-generated

23 SOs trigger spindles in the thalamus that are projected back to neocortex. Here,

24 we used direct intrathalamic recordings from human epilepsy patients to test this

25 canonical interplay. We show that SOs in the anterior thalamus precede

26 neocortical SOs, whereas concurrently-recorded SOs in the mediodorsal

27 thalamus are led by neocortical SOs. Furthermore, sleep spindles, detected in

28 both thalamic nuclei, preceded their neocortical counterparts and were initiated

29 during early phases of thalamic SOs. Our findings indicate an active role of the

30 anterior thalamus in organizing the cardinal sleep rhythms in the neocortex and

31 highlight the functional diversity of specific thalamic nuclei in humans. The

32 concurrent coordination of sleep oscillations by the thalamus could have broad

33 implications for the mechanisms underlying memory consolidation.

34

35 
INTRODUCTION

37 The presence and coordinated interplay of slow oscillations (SOs) and sleep spindles 38 hallmarks non-rapid eye movement (NREM) sleep ${ }^{1-3}$. SOs $(\sim 1 \mathrm{~Hz})$ reflect neuronal 39 network alterations of the membrane potential between periods of neuronal silence 40 ('hyperpolarization', i.e., down state) and neuronal excitation (depolarization, i.e., up state)

414,5 . The origin of SOs has been traditionally located to neocortical circuits ${ }^{5,6}$, triggering 42 time windows of excitability and inhibition not only in neocortex but also in the thalamus 43 and the hippocampus ${ }^{5}$. However, recent evidence from animal models casts doubt on 44 the view that SOs are exclusively initiated and orchestrated by neocortical activity and 45 suggest that the thalamus plays a critical role in synchronizing and coordinating SO 46 activity ${ }^{7-12}$.

47 The depolarizing phase of SOs is assumed to initiate the generation of sleep spindles 48 within the thalamic circuitry ${ }^{13}$ : reciprocal interactions between the thalamic reticular 49 nucleus and thalamocortical neurons result in waxing and waning oscillations in the range 50 of 11-16 Hz. Sleep spindles are usually nested towards the excitable up states of 51 neocortical SOs ${ }^{14-16}$, and are also found in the hippocampus, where they are thought to 52 synchronize hippocampal ripples ${ }^{17-19}$.

53 Importantly, this triple-coupling of sleep related oscillations has been suggested to 54 facilitate memory consolidation, by synchronizing neuronal activity across brain regions 55 and relaying memory representations between the hippocampus and neocortical long56 term stores ${ }^{20,21}$. While a plethora of studies bolster the critical role of sleep oscillations 57 and their coordinated interplay for the memory function of sleep ${ }^{3,22}$, the exact neural 58 circuits enabling their complex orchestration across brain areas are less clear, ultimately 59 impeding our understanding of the prime mechanistic vehicle of memory consolidation. 60 This is particularly striking with respect to the neural circuits facilitating memory 61 consolidation in humans, since direct neural recordings from one of the key players, the 62 thalamus, are scarce. The anterior thalamic nuclei (ANT) and the mediodorsal thalamus 63 (MD) have recently taken center-stage as thalamic key areas for different aspects of 64 human memory functions ${ }^{23,24}$, and might therefore also play a key role in coordinating 65 sleep oscillations relevant for memory consolidation. Leveraging the rare opportunity to 66 record intracranial electroencephalography (iEEG) from the human ANT and MD, together 67 with simultaneous scalp electroencephalography (EEG), we investigated the interplay of 68 NREM sleep oscillations within a thalamocortical network that is known to serve memory 69 functions during wake ${ }^{25}$. 
70 We show that SOs in the ANT, but not the MD, lead neocortical SOs. These results

71 undermine the notion of an exclusive generation of SOs in the human neocortex and

72 highlight the functional diversity of specific thalamic nuclei. Sleep spindles in both the ANT

73 and MD preceded neocortical spindles, in line with their thalamic origin ${ }^{26}$. Furthermore,

74 we show that the nesting of sleep oscillations in the thalamus differs from the nesting in

75 neocortex. Thalamic spindles locked to earlier thalamic SO-phases than their neocortical

76 counterparts. 
bioRxiv preprint doi: https://doi.org/10.1101/2021.12.11.471766; this version posted December 12, 2021. The copyright holder for this

preprint (which was not certified by peer review) is the author/funder, who has granted bioRxiv a license to display the preprint in perpetuity. It is made available under aCC-BY-NC-ND 4.0 International license.

78

79

80

81

82

83

84

85

86

87

88

89

90

91

92

a

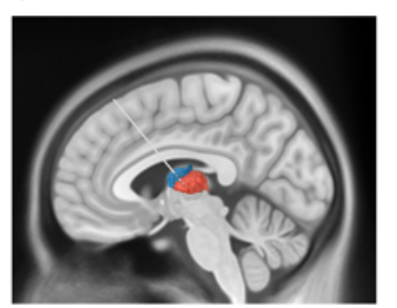

b
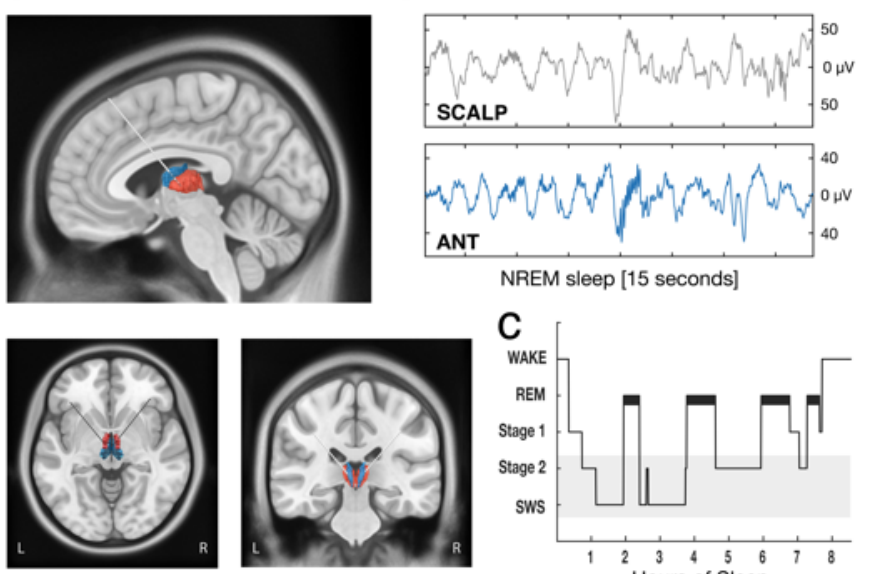

93

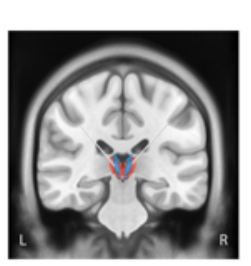

NREM sleep [15 seconds]

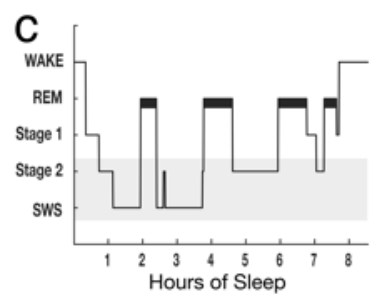

Fig 1. Thalamic electrode placement and sleep architecture. (a) Electrodes were implanted in the left and right ANT (blue) and MD (red). (b) Example of NREM sleep segment (15 sec), comprising SOs and sleep spindles (top row: scalp recording; bottom row: ANT). (c) Hypnogram of a sample participant, showing time spent in different sleep stages across one recording night. 
103

SOs precede neocortical SOs

104 ANT SOs significantly preceded neocortical SOs, as revealed by occurrence probabilities of ANT SOs relative to neocortical SOs (Fig. 2a, significant positive cluster from -0.15 to 0.05 s, $p<0.005$; tested against event-free occurrence probabilities; corrected for multiple comparisons across time). ANT SO occurrence preferentially peaked, on average, briefly before the emergence of neocortical SOs (time of peak: $-0.05 \mathrm{sec}$ ). To further validate this outcome, we specifically determined the phase of ANT SOs for all paired SO-events (i.e., all ANT SOs within $\pm 750 \mathrm{~ms}$ of neocortical SOs) at the time of neocortical SO down state (i.e., thalamo-cortical SO phase-phase coupling). We found significant nonuniform distributions in each of the ANT contacts $(12 / 12)(p<0.05$, corrected for multiple comparisons using the False Discovery Rate (FDR) ${ }^{30}$; Rayleigh test, mean vector length: $0.46 \pm 0.05)$. Moreover, we found a significant nonuniform distribution across contacts (Rayleigh $z=10.95, p<0.0001$ ), with the phase of ANT SOs being ahead of their neocortical counterparts (the phase of neocortical SO down states corresponds to $\pm \pi$; mean coupling direction: $-176.39 \pm 4.94^{\circ}$; see Fig. $2 b$ ).

To showcase the generic features of ANT and neocortical detected SOs, time-frequency representations (TFRs) of SO-locked neocortical (Fig. 2c) and ANT (Fig. 2e) activity were contrasted against event-free events. TFRs time-locked to scalp-derived SO down states exhibited the prototypical modulation of low frequency $(<5 \mathrm{~Hz})$ and spindle power (11$16 \mathrm{~Hz}$ ) during SO events (Fig. 2c). In particular, low frequency power peaked before the SO down state. In contrast, spindle power was diminished during the down state, increased during the positive deflections of the $\mathrm{SO}$ and peaked during the $\mathrm{SO}$ up state (significant clusters: $p<0.05$, corrected for multiple comparisons across time and frequency). Remarkably, while low frequency power for ANT derived SOs showed similar pre-down state increases, power in the spindle band (11-16 Hz) and beyond (> $20 \mathrm{~Hz})$ showed an early power increase tightly locked to the ANT SO down state (Fig. 2e; p < 0.05 , corrected for multiple comparisons across time and frequency). This pattern deviates from generic modulations of spindles by SOs as described in our and previous scalp EEG recordings, where spindles nest towards SO up states ${ }^{1,16}$. Figure 2 d illustrates the scalp EEG grand average, locked to the minimum of neocortical SO down states. Figure $2 f$ illustrates the ANT grand average locked to the minimum of ANT SO down states. 


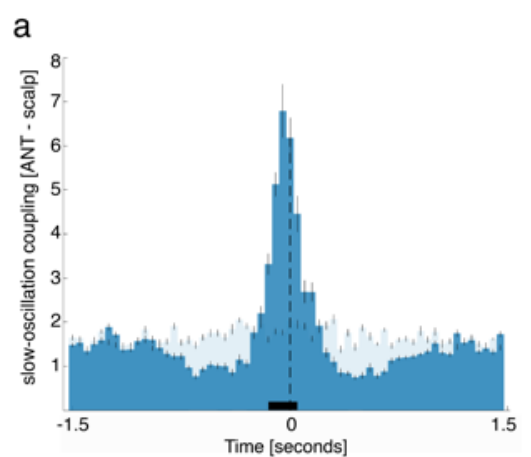

C

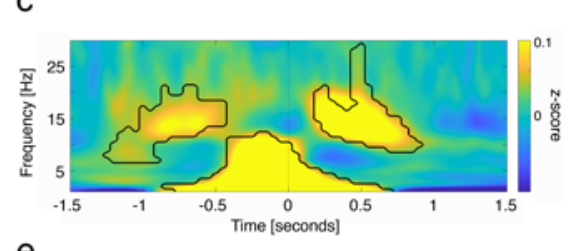

e

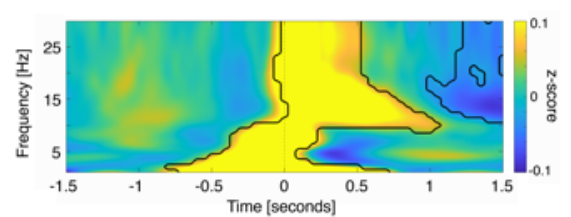

b

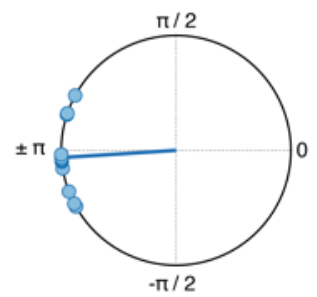

d

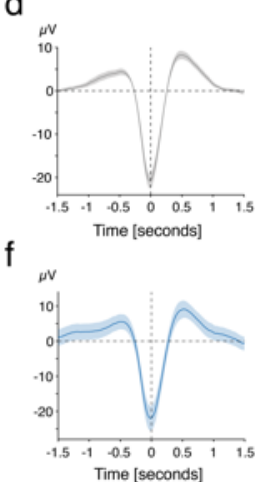

Fig 2. | ANT SOs precede neocortical SOs. (a) Occurrence probabilities of ANT SO down state peaks relative to neocortical SO down state peaks (time = 0; dashed line), indicating that ANT SOs precede neocortical SOs. The solid black bar indicates significant time bins, resulting from comparison with SO-free control events (significant positive cluster from -0.15 to $0.05 \mathrm{sec}, \mathrm{p}<0.005$; time of peak: $-0.05 \mathrm{sec}$ ). (b) Phase of ANT SOs at the time of neocortical SO downstate for paired SO-SO events, illustrating that ANT SO phases preceded their neocortical counterparts (phase of neocortical SO down states corresponds to $\pm \pi$; mean coupling direction: $-176.39 \pm 4.94^{\circ}$; Rayleigh test: $p<0.0001 ; z=10.95$ ). (c) Time-frequency representation of neocortical SOs (locked to neocortical SO down states), contrasted against event-free segments. The contour lines indicate significant clusters $(p<0.05$, corrected for multiple comparisons across time and frequency). (d) Grand average EEG trace of neocortical SOs (mean \pm SEM, negative peak, time 0). (e) Time-frequency representation of all ANT SOs (locked to ANT SO down states), contrasted against event-free segments. The contour lines indicate significant clusters $(p<0.05$, corrected for multiple comparisons across time and frequency). (f) Grand average iEEG trace of ANT SOs (mean \pm SEM, negative peak, time 0).

138 Neocortical SOs precede SOs in the MD

139 Next, we asked, whether SOs leading neocortical SOs is specific to the ANT or can also

140 be found in other thalamic nuclei. To address this question, we investigated the interplay

141 between neocortical SOs and SOs in the mediodorsal thalamus (MD).

142 In stark contrast to ANT SOs, SOs in the MD did not precede, but were on average led

143 by neocortical SOs, as evidenced by occurrence probabilities (Fig. 3a; significant positive

144 cluster from -0.05 to $0.1 \mathrm{~s}, \mathrm{p}=0.021$; corrected for multiple comparisons across time;

145 time of peak: $0.05 \mathrm{sec}$ ). Again, we determined the phase of thalamic SOs for all paired

146 SO-events at the time of neocortical SO down state ( $\pm 750 \mathrm{~ms})$. We found significant 147 nonuniform distributions in all MD contacts (15/15, $p<0.05$, corrected for multiple 148 comparisons using FDR; Rayleigh test, mean vector length: $0.41 \pm 0.06$ ) and a significant 149 nonuniform distribution across contacts (Rayleigh $z=7.94, p=0.0001$ ), with the phase 
150 of MD SOs following their neocortical counterparts (mean coupling direction: $133.51 \pm$ 151 10.91'; see Fig. 3b). TFRs time-locked to scalp-derived SO down states exhibited the 152 typical modulation of low frequency $(<5 \mathrm{~Hz})$ and spindle power $(11-16 \mathrm{~Hz})$ during SO 153 events (Fig. 3c; $p<0.05$, corrected for multiple comparisons across time and frequency).

154 Similar to the ANT, TFRs locked to the down states of MD SOs exhibited significant low 155 frequency power increases before the down state-peak (Fig. 3e, time = 0), and power 156 increases in the sleep spindle band and beyond locked to the down state (significant 157 cluster: $p<0.05$, corrected for multiple comparisons across time and frequency). Figure $1583 d$ illustrates the scalp EEG grand average, locked to the minimum of neocortical SO 159 down states. Figure $3 f$ illustrates the MD grand average locked to the minimum of MD 160 SO down states.

161 Finally, we directly compared ANT-neocortical and MD-neocortical interactions with 162 regards to SOs and tested whether ANT SOs would systematically emerge earlier (in 163 relation to neocortical SOs) than MD SOs. The occurrence probabilities of ANT SOs 164 relative to neocortical SOs differed significantly from those obtained from MD SOs relative 165 to neocortical SOs, with ANT SOs preferentially emerging before MD SOs (Fig 3g; 166 significant positive cluster from -0.25 to $-0.05 \mathrm{~s}, \mathrm{p}=0.018$; corrected for multiple 167 comparisons across time; for a direct comparison of ANT-MD SOs see Supplementary

168 Fig. 1). Also, the phase distribution for the preferential coupling between thalamus and 169 neocortex differed significantly when comparing ANT and MD related SO coupling (Fig. 170 3h; Watson-Williams test: $F=12.7, p=0.0015)$, supporting the leading role of ANT SOs 171 as compared to the MD. 
bioRxiv preprint doi: https://doi.org/10.1101/2021.12.11.471766; this version posted December 12, 2021. The copyright holder for this preprint (which was not certified by peer review) is the author/funder, who has granted bioRxiv a license to display the preprint in perpetuity. It is made available under aCC-BY-NC-ND 4.0 International license.

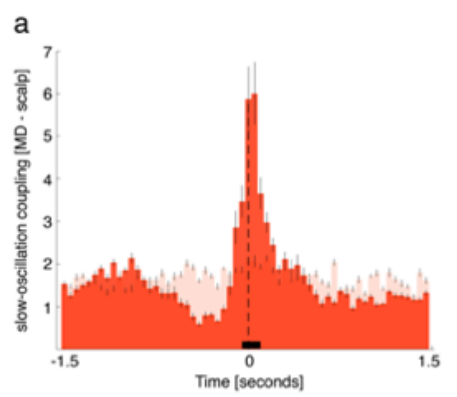

C

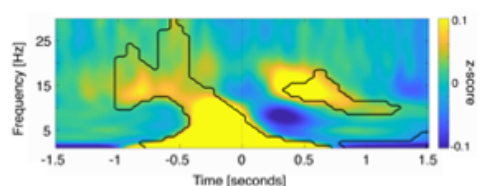

e

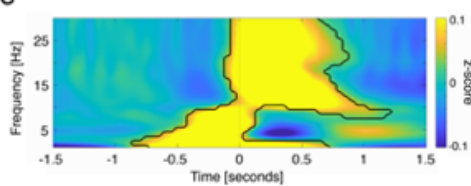

g

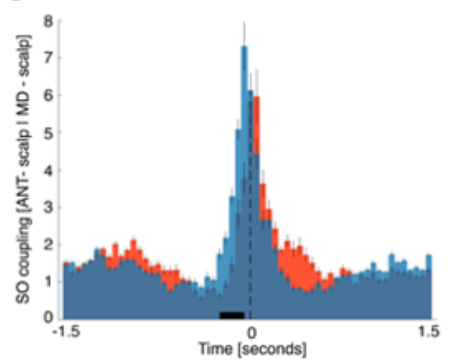

b

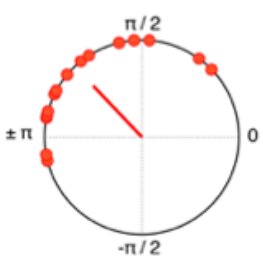

d
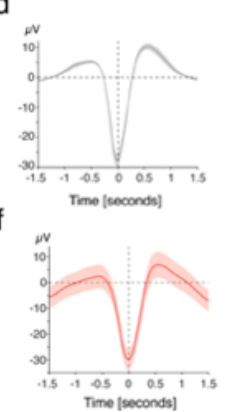

h

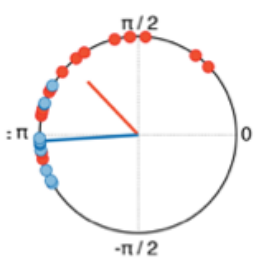

172

Fig 3. | Neocortical SOs precede SOs in the MD. (a) Occurrence probabilities of MD SO down state peaks relative to neocortical SO down state peaks (time $=0$, dashed line), illustrating that, on average, neocortical SOs lead MD SOs. The solid black line indicates significant time bins, resulting from comparison with SO-free control events (significant positive cluster from -0.05 to $0.1 \mathrm{sec}, \mathrm{p}=0.021$; time of peak: $0.05 \mathrm{sec}$ ). (b) Phase of MD SOs at the time of neocortical SO downstate for paired SO-SO events, depicting that MD SO phases followed their neocortical counterparts (phase of neocortical SO down states corresponds to $\pm \pi$; mean coupling direction: $133.51 \pm 10.91^{\circ}$; Rayleigh test: $p<0.0001 ; z=7.94$ ). (c) Time-frequency representation of neocortical SOs (locked to neocortical SO down states), contrasted against event-free segments. The contour lines indicate significant clusters $(p<0.05$, corrected for multiple comparisons across time and frequency). (d) Grand average EEG trace of neocortical SOs (mean \pm SEM, negative peak, time 0). (e) Time-frequency representation of all MD SOs (locked to MD SO down states), contrasted against event-free segments. The contour lines indicate significant clusters $(p<0.05$, corrected for multiple comparisons across time and frequency). (f) Grand average iEEG trace of MD SOs (mean \pm SEM, negative peak, time 0). (g) Occurrence probabilities for the direct comparison of ANT-neocortical and MD-neocortical SO interactions, showing that ANT SOs preferentially emerge before MD SOs with regards to their neocortical counterparts (significant positive cluster from -0.25 to $-0.05 \mathrm{sec}, \mathrm{p}=0.018$; corrected for multiple comparisons across time;). (h) The phase distribution for the coupling between thalamus and neocortex differed significantly when comparing ANT and MD related SO coupling (Watson-Williams test: $F=12.7, p=0.0015$ ). 
183

\section{4}

ANT spindles precede neocortical spindles

185

Animal models established that sleep spindles are generated in the thalamus and spread

186 to the neocortex along thalamocortical fibers ${ }^{26}$. However, it is unclear which of the many thalamic nuclei contribute to the generation of spindles and whether this translates to humans. Here, we show that the human ANT is putatively part of the spindle generating thalamic circuit, as ANT spindle occurrence peaked on average before the emergence of

1900.15 to $0.2 \mathrm{~s}, \mathrm{p}=0.019$; corrected for multiple comparisons across time; time of peak: -

$1910.1 \mathrm{sec})$.

192 We further assessed the interplay of ANT and neocortical sleep spindles using a 193 complementary analytical approach. Specifically, we tested to what extend spindle194 related oscillatory power-modulations would precede those in the neocortex, by 195 computing power-power correlations in the sleep spindle band (see methods for details).

196 We found a significant, off-diagonal cluster of temporal cross-correlations with regards 197 to spindle power between ANT and neocortical sites ( $p=0.004$, cluster corrected across 198 time; Fig. 4b), supporting our initial finding that ANT spindles lead their neocortical 199 counterparts. Figure 4c and e depict the spindle-locked (time $0=$ maximally negative 200 amplitude) neocortical (Fig. 4c) and ANT (Fig. 4e) related TFRs. While the power of scalp 201 derived spindles was confined to the classical sleep spindle range ( 11-16 Hz; Fig. 4c), 202 power increases in the spindle band transitioned seamlessly into higher frequencies (> $20320 \mathrm{~Hz}$ ) in case of ANT recordings (Fig. 4e; $p<0.05$, corrected for multiple comparisons 204 across time and frequency). Figure $4 d$ and $f$ illustrate the grand average 205 electrophysiological traces across scalp electrodes / ANT contacts (mean \pm SEM, 206 respectively), locked to the neocortical (Fig. 4d) and ANT (Fig. 4f) sleep spindle peak 207 (maximally negative amplitude, time 0). Figure 4d illustrates the scalp EEG grand average, 208 locked to the neocortical sleep spindle peak. Figure $4 \mathrm{f}$ illustrates the ANT grand average, 209 locked to the ANT sleep spindle peak. 


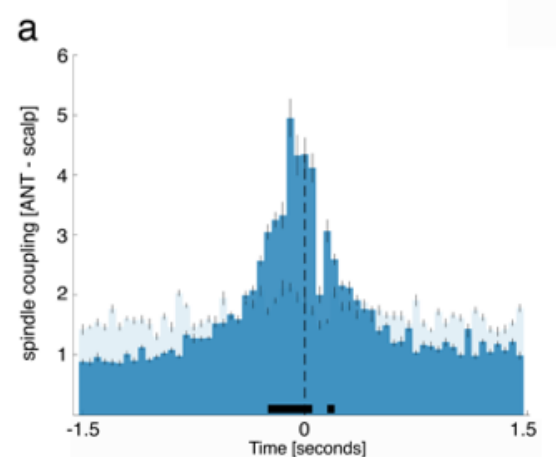

C

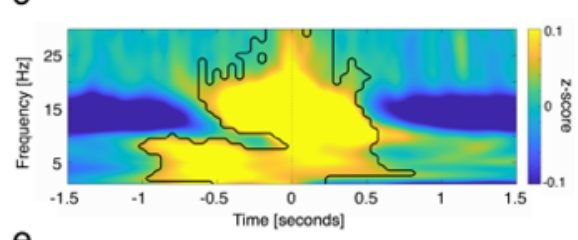

e

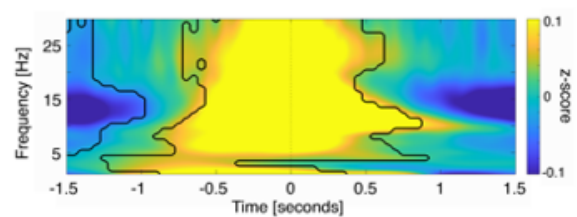

b

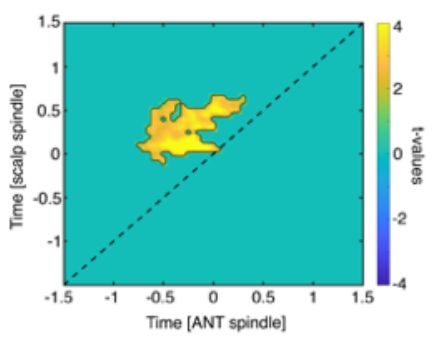

d

f
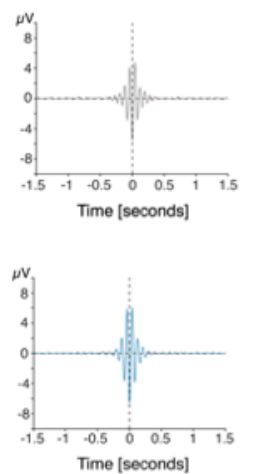

212

Fig 4. | ANT spindles lead neocortical spindles. (a) Occurrence probabilities of ANT spindle peaks relative to neocortical spindle peaks (maximal negative amplitude, time $=0$; dashed line), indicating that ANT spindles precede neocortical spindles. The solid black line indicates significant time bins, resulting from comparison with spindle-free control events (significant positive clusters from -0.25 to $-0.05 \mathrm{sec}, \mathrm{p}<0.005$ \& 0.15 to $0.2 \mathrm{sec}, \mathrm{p}=0.019$; corrected for multiple comparisons across time; time of peak: $-0.1 \mathrm{sec}$ ). (b) Power-power correlations in the sleep spindle band for paired spindle events (locked to neocortical spindle peak), contrasted against event-free correlation maps. The contour lines indicate the significant cluster ( $p=0.004$, cluster corrected across time), depicting that ANT related spindle power precedes neocortical spindle power. (c) Time-frequency representation of neocortical spindles (locked to neocortical spindle peak), contrasted against event-free segments. The contour lines indicate significant clusters ( $p$ $<0.05$, corrected for multiple comparisons across time and frequency). (d) Grand average EEG trace of neocortical spindles (mean \pm SEM, maximally negative amplitude, time 0). (e) Time-frequency representation of all ANT spindles (locked to ANT spindle peak), contrasted against event-free segments. The contour lines indicate significant clusters ( $p<0.05$, corrected for multiple comparisons across time and frequency). (f) Grand average iEEG trace of ANT spindles (mean \pm SEM, maximally negative amplitude, time 0). 


\section{MD spindles lead neocortical spindles}

228 Next, we tested the temporal relationship between MD and neocortical spindles. MD

229 spindles occurred on average before neocortical spindles (Fig. 5a; significant positive

230 clusters: -0.3 to $0.05 \mathrm{~s}, \mathrm{p}<0.005$; corrected for multiple comparisons across time; time

231 of peak: $-0.05 \mathrm{sec}$ ). We also assessed power-power correlations in the sleep spindle

232 band for neocortical- MD paired spindle events (locked to neocortical spindle peaks). We

233 found a significant, off-diagonal cluster of temporal cross-correlation with regards to

234 spindle power between MD and neocortical sites (Fig. 5b; tested against event-free

235 segments; $p=0.002$, cluster corrected across time), indicating that MD spindle-related

236 power modulations preceded neocortical spindle-related power.

237 Fig. 5c and e depict the spindle-locked (maximally negative amplitude) neocortical and

238 MD related TFRs. Power of scalp derived spindles was, as expected, confined to the

239 classical sleep spindle range $(\sim 11-16 \mathrm{~Hz}$; Fig. 5c), while TFRs related to MD spindles

240 exhibited power increases in the spindle band and beyond (>20 Hz). Overall, the

241 relationship between $\mathrm{MD}$ spindles and neocortical spindles was highly similar to the

242 relationship between ANT spindles and neocortical spindles (see Figs. 4a + b). Figure 5d

243 illustrates the scalp EEG grand average, locked to the neocortical sleep spindle peak.

244 Figure $5 f$ illustrates the MD grand average, locked to the MD sleep spindle peak. Figure

$2455 \mathrm{~g}$ shows a comparison of ANT-neocortical and MD-neocortical interactions with regards

246 to spindles. The timing of ANT and MD-related spindles in relation to neocortical spindles

247 was highly similar. No significant differences emerged (cluster with lowest $p=0.52$ ). 
a

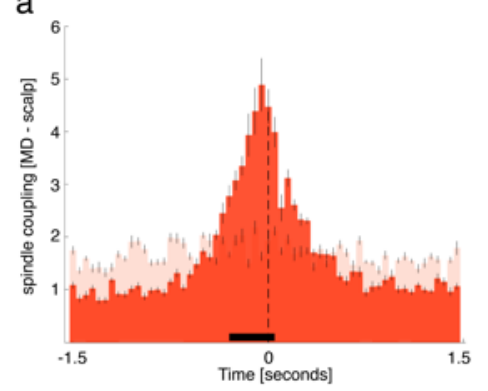

C

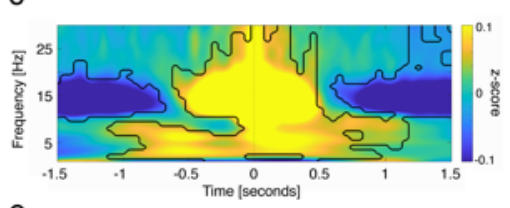

e

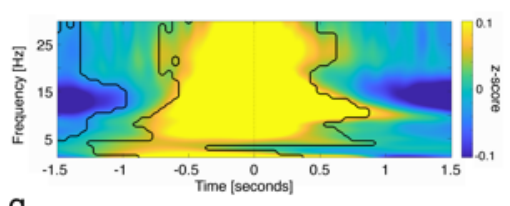

g

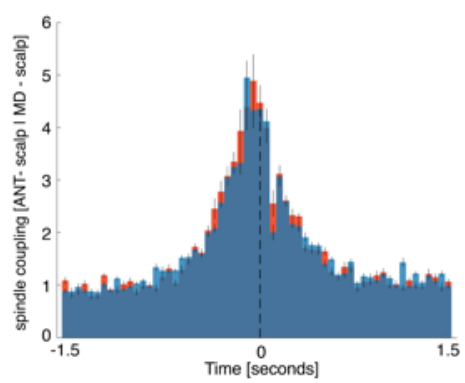

b

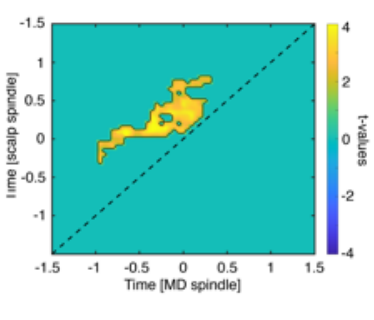

d

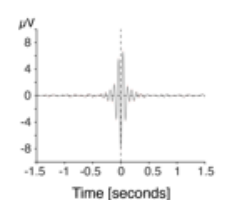

f

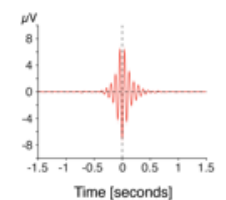

nime [seconds]

Fig 5. | MD spindles lead neocortical spindles. (a) Occurrence probabilities of MD spindle peaks relative to neocortical spindle peaks (maximal negative amplitude, time $=0$; dashed line), indicating that MD spindles precede neocortical spindles. The solid black line indicates significant time bins, resulting from comparison with spindle-free control events (significant positive cluster from -0.3 to $0.05 \mathrm{sec}, \mathrm{p}<0.005$; corrected for multiple comparisons across time; time of peak: $-0.05 \mathrm{sec}$ ). (b) Power-power correlations in the sleep spindle band for paired spindle events (locked to neocortical spindle peak), contrasted against event-free correlation maps. The contour lines indicate the significant cluster ( $p=0.002$, cluster corrected across time), showing that MD related spindle power precedes neocortical spindle power. (c) Time-frequency representation of neocortical spindles (locked to neocortical spindle peak), contrasted against event-free segments. The contour lines indicate significant clusters $(p<0.05$, corrected for multiple comparisons across time and frequency). (d) Grand average EEG trace of neocortical spindles (mean \pm SEM, maximally negative amplitude, time 0). (e) Time-frequency representation of all MD spindles (locked to MD spindle peak), contrasted against event-free segments. The contour lines indicate significant clusters $(p<0.05$, corrected for multiple comparisons across time and frequency). (f) Grand average iEEG trace of MD spindles (mean \pm SEM, maximally negative amplitude, time 0). (g) Direct comparison of ANT-neocortical and MD-neocortical interactions with regards to spindles yielded that the timing of ANT and MD-related spindles in relation to neocortical spindles was highly similar (cluster with lowest $\mathrm{p}=0.52$ ).

251 So far, we established that both, neocortical SOs and spindles are preceded by corresponding activity in the ANT. These results also imply that the properties of local SO-spindle interactions, which are thought to shape memory consolidation ${ }^{14,31}$, may 
256 in ANT as well as scalp recordings and extracted the onset latencies of spindles relative

257 to their corresponding SO down states within both regions, respectively.

258 In line with previous findings ${ }^{19,32}$, occurrence probabilities of neocortical spindles with 259 respect to neocortical SOs (Fig. 6a), indicated that spindles started specifically at the SO 260 down-to-up transition (0.05 to $0.15 \mathrm{~s} ; \mathrm{p}<0.0001$; peak: $0.1 \mathrm{sec}$ ). In contrast, ANT spindles preferential emerged around ANT SO down states (-0.15 to $0.25 \mathrm{~s} ; \mathrm{p}=0.0012$; peak: 0 s; Fig. 6b). These diverging dynamics also became apparent when depicting the SO-locked (down state) neocortical and ANT derived SO-spindle TFRs (Fig. 6c: locked to neocortical SO down state; Fig. 6d: locked to ANT SO down state). TFRs for both ANT and scalp derived SO-spindle complexes exhibited characteristic power-increases in the SO and sleep spindle band $(p<0.05$, corrected for multiple comparisons across time and frequency). However, while neocortical spindles showed their typical power peaks well after the corresponding down states ${ }^{1,16}$, increases in the ANT sleep spindle band were initiated around the ANT SO down states.

270 Finally, directly comparing the occurrence probabilities of thalamic and neocortical SO271 spindle complexes affirmed that ANT spindles locked significantly earlier to SOs than 272 neocortical spindles (-0.3 to $-0.1, p=0.0039$; Fig. 6e). In a complementary analytical 273 approach, we determined the preferred phase of SO-spindle modulation (i.e., SO-spindle 274 coupling), for both ANT and neocortical events, respectively, by assessing the SO phases 275 corresponding to the spindle onset latencies in each contact / scalp electrode. In 12/12 276 ANT contacts we found significant nonuniform distributions $(p<0.05$, corrected for 277 multiple comparisons using FDR; Rayleigh test, mean vector length: $0.41 \pm 0.03$ ) and a 278 significant nonuniform distribution across contacts (Rayleigh $z=9.33, p<0.0001$ ), with 279 spindles starting near the SO down state (corresponding to $\pm 180^{\circ}$; mean coupling 280 direction: $-175.22^{\circ} \pm 6.92^{\circ}$; see Fig. $6 f$ ). Similarly, we found in $7 / 8$ scalp contacts a 281 significant nonuniform distribution $(p<0.05$, corrected for multiple comparisons using 282 FDR; Rayleigh test, mean vector length: $0.27 \pm 0.02)$. Again, a significant nonuniform 283 distribution across participants was present (Rayleigh $z=6.91, p<0.0001$ ), with spindles 284 preferentially starting at the SO down-to-up transition (mean coupling direction: -137.14 $285 \pm 7.58^{\circ}$; see Fig. 6f). Next, we tested whether the preferred coupling phases would vary 286 systematically between ANT and neocortical areas, using the circular Watson-Williams 287 test. Indeed, we found a significant difference in relation to the preferred phase of ANT 288 and neocortical SO-spindle modulation ( $F=9.31 ; p=0.0069)$, corroborating the finding 289 that spindles in the thalamus lock to earlier phases of the SO. Finally, we quantified the 290 directional influence of SOs on spindles in both ANT and neocortical data, using the 
bioRxiv preprint doi: https://doi.org/10.1101/2021.12.11.471766; this version posted December 12, 2021. The copyright holder for this preprint (which was not certified by peer review) is the author/funder, who has granted bioRxiv a license to display the preprint in perpetuity. It is made available under aCC-BY-NC-ND 4.0 International license.

a

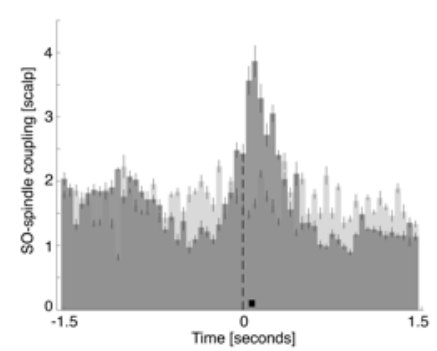

C

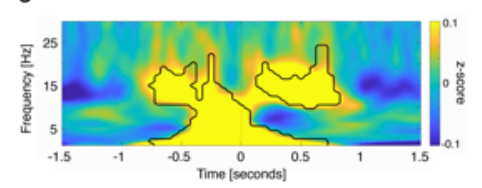

e

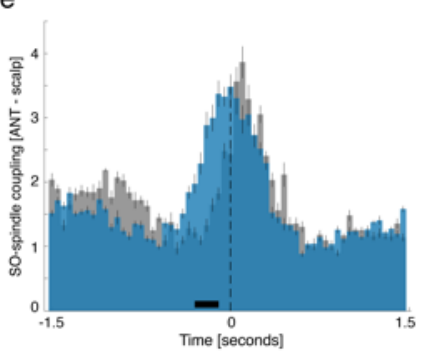

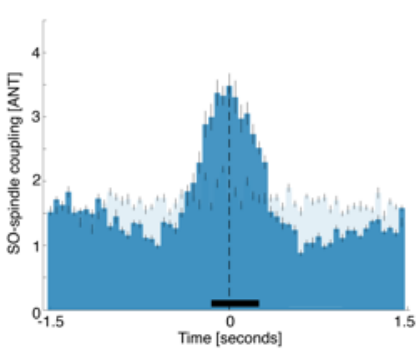

d

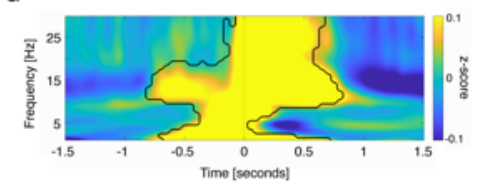

f

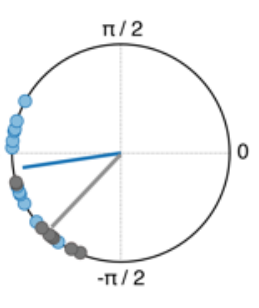

Fig 6. | ANT spindles lock to early SO phases. (a) Occurrence probabilities of neocortical spindle onsets with respect to neocortical SO down states, illustrating that spindles started specifically at the SO down-to-up transition (0.05 to 0.15 sec; $p<0.0001$; peak: $0.1 \mathrm{sec}$ ). (b) ANT spindles preferential emerged around ANT SO down states (-0.15 to $0.25 \mathrm{sec} ; \mathrm{p}=0.0012$; peak: $0 \mathrm{sec}$ ). (c) Time-frequency representation of neocortical SO-spindle events (locked to neocortical SO down state peaks), contrasted against event-free segments. The contour lines indicate significant clusters ( $p<0.05$, corrected for multiple comparisons across time and frequency). (d) Time-frequency representation of all ANT SO-spindle events (locked to ANT SO down state peaks), contrasted against event-free segments. The contour lines indicate significant clusters ( $p<0.05$, corrected for multiple comparisons across time and frequency). (e) Direct comparison of the occurrence probabilities of ANT and neocortical SO-spindle complexes yielded that ANT spindles locked significantly earlier to SOs as compared to neocortical spindles $(-0.3$ to $-0.1, p=0.0039)$. (f) Phases of the SO-spindle modulation derived from neocortical (gray circles) and ANT (blue circles) events. Neocortical spindle onsets preferentially emerged at the neocortical SO down-to-up transition (phase of neocortical SO down states corresponds to $\pm \pi$; mean coupling direction: $-137.14 \pm 7.58^{\circ}$; Rayleigh $z=6.91, p<0.0001$ ), while ANT spindles started specifically around the ANT SO down states (mean coupling direction: $-175.22^{\circ} \pm 6.92^{\circ}$; Rayleigh z = 9.33, $\mathrm{p}<0.0001$ ). The preferred phases of SO-spindle modulation differed significantly between the ANT and neocortical sites (Watson-Williams test: $F=9.31$; $p=0.0069)$. (g) Directional SO-spindle coupling as obtained by the phase-slope index (PSI; mean \pm SEM) for ANT (blue circles) and neocortical (gray circles) SO-spindle complexes. SO-phases significantly predicted spindle amplitudes both in case of ANT (t-test against zero: $p=0.037$ ) and neocortical SOspindle events (t-test against zero: $p=0.044)$. 
MD spindles lock to early SO phases

302 We have shown that neocortical SOs precede MD SOs, while MD spindles lead 303 neocortical spindles. Accordingly, the coupling within MD related SO-spindle complexes 304 is expected to be shifted compared to neocortical SO-spindle complexes. Occurrence 305 probabilities indicated that neocortical spindles started specifically at the down-to-up transition of neocortical SOs (0.1 to $0.3 \mathrm{~s} ; \mathrm{p}=0.003$; peak: $0.1 \mathrm{~s}$; Fig 7a). MD spindles, however, started preferentially around the MD SO down states (-0.35 to $0 \mathrm{~s} ; \mathrm{p}<0.0001$; peak: -0.05 s; Fig 7b). Figures 7c and d show the neocortical and MD SO-spindle TFRs

309 (Fig. 7c: locked to neocortical SO down states; Fig. 7d: locked to MD SO down state).

310 TFRs for both scalp and MD derived SO-spindle complexes featured power-increases in

311 the SO and sleep spindle band ( $p<0.05$, corrected for multiple comparisons across time 312 and frequency). Neocortical spindles exhibited their typical power peaks after the 313 corresponding down states, while power peaks of MD sleep spindles started around MD

314 SO down states. Accordingly, the direct comparison of occurrence probabilities revealed 315 that MD spindles locked significantly earlier to accompanying MD SOs as did neocortical 316 spindles to neocortical SOs (-0.35 to 0.05, $p<0.0001$; Fig. 7e).

317 Next, we determined the preferred phase of SO-spindle modulation for MD and 318 neocortical events, respectively, by assessing the SO phases corresponding to the 319 spindle onset latencies in each contact / scalp electrode. In 15/15 thalamic contacts, we 320 found significant nonuniform distributions $(p<0.05$, corrected for multiple comparisons 321 using FDR; Rayleigh test, mean vector length: $0.42 \pm 0.03)$. Also, across contacts the 322 distribution was significantly nonuniform (Rayleigh $z=12.10, p<0.0001$ ), with spindles 323 starting near the SO down state (corresponding to $\pm 180^{\circ}$; mean coupling direction: $324172.26^{\circ} \pm 6.67^{\circ}$; see Fig. $7 f$.

325 For scalp derived data, we found in 9/11 contacts a significant nonuniform distribution ( $p$ $326<0.05$, corrected for multiple comparisons using FDR; Rayleigh test, mean vector length: $3270.21 \pm 0.02)$. Again, a significant nonuniform distribution across participants was present 328 (Rayleigh $z=8.18, p<0.0001$ ), with spindles preferably starting at the down-to-up 329 transition of neocortical SOs (mean coupling direction: $-138.43 \pm 9.05^{\circ}$; see Fig. $7 f$ ). 330 When directly comparing the preferred coupling phases between MD and neocortical 331 areas, we found a significant difference (Watson-Williams test: $F=17.33 ; p=0.0003$ ), 332 supporting the outcome that spindles in the MD start at earlier phases of the SO then 333 neocortical spindles. We also quantified the directional influence of SOs on spindles in 334 both $\mathrm{MD}$ and neocortical data, using the phase-slope index ${ }^{34,35}$. Again, we show that 335 SOs predicted sleep spindle activity within MD and neocortex, respectively, as evidenced 
bioRxiv preprint doi: https://doi.org/10.1101/2021.12.11.471766; this version posted December 12, 2021. The copyright holder for this preprint (which was not certified by peer review) is the author/funder, who has granted bioRxiv a license to display the preprint in perpetuity. It is made available under aCC-BY-NC-ND 4.0 International license.

by a positive PSI (MD: $0.0016 \pm 0.0006$; t-test against zero: $t=2.36, p=0.032$; neocortex: $0.0028 \pm 0.002$; t-test against zero: $t=2.23, p=0.049$; Fig. $7 g$ ). Finally, we compared the features of ANT- and MD-derived SO-spindle complexes. While MD

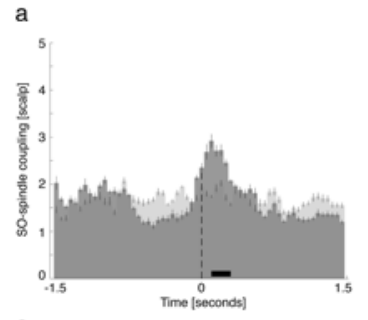

c

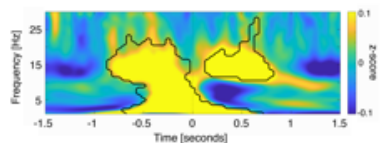

$e_{5}$

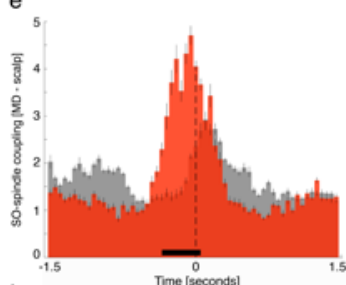

$\mathrm{h}$

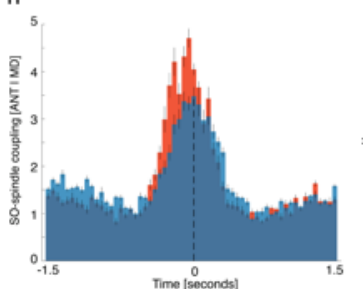

b
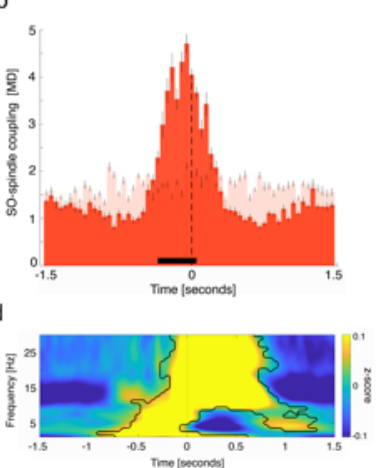

$\mathrm{g}$
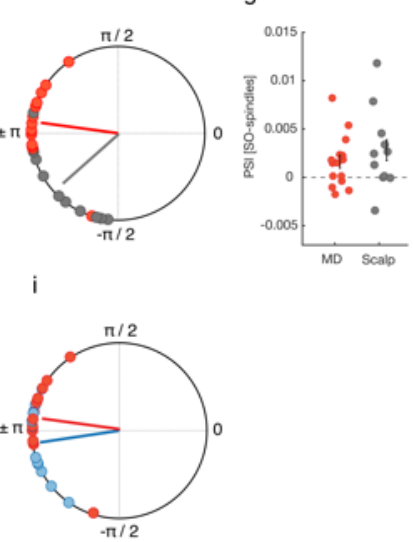

Fig7. | MD spindles lock to early SO phases. (a) Occurrence probabilities of neocortical spindle onsets with respect to neocortical SO down states, indicating that spindles started specifically at the SO down-to-up transition (0.05 to $0.15 \mathrm{sec} ; \mathrm{p}<0.0001$; peak: $0.1 \mathrm{sec}$ ). (b) MD spindles started around MD SO down states (-0.35 to 0 sec; $p<0.0001$; peak: $-0.05 \mathrm{sec}$ ). (c) Time-frequency representation of neocortical SO-spindle events (locked to neocortical SO down state peaks), contrasted against event-free segments. The contour lines indicate significant clusters $(p<0.05$, corrected for multiple comparisons across time and frequency). (d) Time-frequency representation of all MD SOspindle events (locked to MD SO down state peaks), contrasted against event-free segments. The contour lines indicate significant clusters $(p<0.05$, corrected for multiple comparisons across time and frequency). (e) Direct comparison of the occurrence probabilities of $\mathrm{MD}$ and neocortical SO-spindle complexes revealed that MD spindles start at significantly earlier SO phases as compared to neocortical spindles $(-0.35$ to $0.05, p<0.0001)$. (f) Phases of the SO-spindle modulation derived from neocortical (gray circles) and MD (red circles) events. Neocortical spindle onsets preferentially emerged at the neocortical SO down-to-up transition (phase of neocortical SO down states corresponds to $\pm \pi$; mean coupling direction: $-138.43 \pm 9.05^{\circ}$; Rayleigh $z=8.18, p<0.0001$ ), while MD spindles started specifically around the MD SO down states (mean coupling direction: $172.26 \pm 6.67^{\circ}$; Rayleigh $z=12.10, p$ $<0.0001$ ). The preferred phases of SO-spindle modulation differed significantly between the MD and neocortical sites (Watson-Williams test: $F=17.33 ; p=0.0003$ ). (g) Directional SO-spindle coupling obtained by the phase-slope index (PSI; mean \pm SEM) for MD (red circles) and neocortical (gray circles) SO-spindle complexes. SO-phases significantly predicted spindle amplitudes both in case of MD (t-test against zero: $p=0.032$ ) and neocortical SO-spindle events (t-test against zero: $\mathrm{p}=0.049)$. $(\mathrm{h}+\mathrm{i}$ ) Directly comparing ANT- and MD-derived SO-spindle complexes did not yield a significant difference $(h)$ occurrence probabilities: $p=0.11$; (i) phase distribution: $F=1.29 ; p=0.25$ ). 


\section{DISCUSSION}

344 Our results demonstrate a leading role of anterior thalamic activity in the orchestration of

345 cardinal sleep rhythms in humans. In particular, we found that SOs recorded in the human

346 ANT preceded their neocortical counterparts, in contrast to SOs recorded in the MD,

347 which were led by neocortical SOs. Spindles recorded in ANT as well as MD preceded

348 neocortical spindles. Finally, both in the ANT and the MD, thalamic spindles locked to

349 early SO-phases, facilitating the prototypical synchrony between SOs and spindles in the 350 neocortex.

351 Sleep oscillations have gained considerable interest over the last decade, mainly due their presumed role in facilitating the memory function of sleep ${ }^{22}$. SOs are thought to represent

353 the time-giving pacemakers of memory consolidation and have been studied extensively

354 in both rodent models and during human sleep ${ }^{36,37}$. Traditionally, they have been 355 assumed to be solely generated within neocortical circuits ${ }^{5,6}$. However, recent animal 356 studies cast doubt on this view by identifying essential thalamic contributions for SO 357 generation and coordination ${ }^{7-12}$. Thalamic neurons have been shown to exhibit intrinsic, 358 rhythmic up and down states in isolated conditions (i.e., without neocortical inputs ${ }^{7,38}$ ), 359 while phasic burst-firing of thalamocortical neurons at the onset of up states often 360 precedes the firing of neocortical neurons by $20-50 \mathrm{~ms}^{9,39,40}$. Optogenetic stimulation 361 of thalamocortical neurons efficiently initiates neocortical up states ${ }^{8,41}$. In addition, 362 thalamic activity terminates SO up states synchronously across neocortical areas, thus 363 mediating a well-controlled down state transition ${ }^{42}$. In sum, these findings suggest that 364 thalamic activity exerts a controlling influence on neocortical SOs, by coordinating up and 365 down state dynamics in neocortical circuits. However, the generalization of thalamic 366 contributions, and specifically of distinct thalamic nuclei, to sleep oscillations has been 367 shown to be challenging across species (e.g., ${ }^{43,44}$ ), prompting their direct assessment 368 during human sleep.

369 To investigate potential contributions of the ANT to neocortical SO coordination, we 370 detected SOs during NREM sleep in intracranial contacts located in the ANT as well as 371 scalp EEG. We show that the ANT not only exhibits a slow oscillatory rhythm (note that 372 the ANT signal is likely to be of local origin due to a bipolar montage), but, importantly, 373 that ANT SOs precede their neocortical counterparts. This might indicate that the ANT 374 guides SO activity in the neocortex. Focusing on paired events only (i.e., time-windows 375 where both neocortical and ANT SOs were present), we found that ANT SO phases 376 significantly lead neocortical SO phases. Notably, this pattern of results was specific to 377 ANT recordings. SOs in the MD did not precede but rather followed neocortical SOs, 
which is in line with a previous report of neocortical SOs leading SOs in the human pulvinar ${ }^{15}$. Our findings thus highlight both a specific role of the ANT for SO dynamics and, more generally, the functional diversity of specific thalamic nuclei in humans ${ }^{45}$.

381 What makes the ANT a favorable candidate for sculpting neocortical SOs? The ANT is a key node in the limbic circuit ${ }^{46}$ and functionally relevant for human memory ${ }^{24,25}$. It has extensive connections with the anterior cingulate, orbitofrontal cortex and the hippocampus ${ }^{47-49}$. Notably, medial prefrontal regions, such as the orbitofrontal and the cingulate cortex have been identified as neocortical hot spots for the emergence of SO during human NREM sleep ${ }^{50-52}$. In addition, work in rodent models has shown that midline and anterior thalamic nuclei are likely candidates to influence global neocortical activity during sleep due to their widespread projections 9,42. Hence, the dense connectivity of the ANT with medial prefrontal regions and within the limbic circuit sets the stage for ANT activity to shape neocortical SOs and coordinate sleep oscillations 391 between regions implicated in memory consolidation.

392 We also found clear evidence for spindles in ANT and MD. Spindles emerge in recurrent 393 loops between the thalamic nucleus reticularis (TRN) and thalamocortical neurons, which 394 in turn forward them to the neocortex and hippocampus ${ }^{26,53}$. There have been conflicting 395 findings across animal species about whether the TRN innervates the ANT and, hence, 396 whether or not spindles are present in the ANT ${ }^{23,43,44,54-58}$. Our results provide a clear 397 picture in humans: spindles are not only present both in the ANT and MD but also 398 precede neocortical spindles, indicating an involvement of both nuclei in spindle 399 generation and their projection to neocortical areas. In line with our interpretation, 400 optogenetic stimulation of the rodent anterodorsal TRN, which innervates the ANT 44,56, 401 has been shown to initiate spindles in the neocortex and hippocampus ${ }^{59}$, indicating that 402 the ANT might synchronize key areas of memory consolidation ${ }^{27}$. With regards to the $403 \mathrm{MD}$ it has been found that its neurons exhibit increased firing during spindle events ${ }^{29}$ in 404 rodents. In humans, reduced MD volume in persons with schizophrenia is associated 405 with dampened neocortical spindle activity ${ }^{60,61}$, further pointing towards a role of the MD 406 in the thalamo-cortical spindle transfer. In sum, our results extend previous research 407 identifying sleep spindles in other human thalamic nuclei ${ }^{15,62}$, by showing that sleep 408 spindles in the ANT and MD precede neocortical spindles during human sleep, indicating 409 a role of both nuclei in thalamo-cortical spindle projections.

$410 \quad$ Finally, we set out to characterize the properties of SO-spindle interactions within 411 the ANT and the MD. Spindles tend to nest within SO up states in scalp EEG recordings $41214,32,63$. Importantly, the precise timing of neocortical spindle peaks with respect to the SO 
413 upstate has been shown to be tightly linked to the behavioral expressions of memory 414 consolidation ${ }^{32,64,65}$. Hence, the characteristics of SO-spindle interactions in the ANT 415 might therefore highlight how the thalamus concomitantly groups SOs and spindles in 416 the neocortex, leading to the well-known oscillatory nesting. We found that spindles in 417 the ANT were initiated around the thalamic SO down state, in contrast to neocortical 418 spindles that preferentially started later, at the neocortical SO down to up state transition 419 (leading to the well-known nesting of neocortical spindles towards the up states) ${ }^{3,63}$.

420 Thus, ANT spindles started at significantly earlier SO phases as compared to neocortical 421 SO-spindle interactions. Notably, this divergent property of SO-spindle coordination is a 422 necessary pre-requisite to allow for a concomitant coordination of neocortical SOs and 423 spindles by the ANT. Specifically, while we show that both ANT SOs and spindles 424 preceded their neocortical counterparts, the time lag between ANT and neocortical 425 spindles exceeded the time lag between ANT and neocortical SOs. Hence, to enable the 426 prototypical SO-spindle modulation in neocortical circuits (i.e., nesting of spindles 427 towards the SO upstate) under the assumption that both graph-elements in the 428 neocortex are governed by the ANT, it is inevitable that spindles start at relatively earlier 429 phases of the SO in the ANT as compared to spindles of neocortical SO-spindle 430 complexes.

431 We found a similar pattern of SO-spindle interactions within the MD, with spindles starting 432 at early phases of the SO. Interestingly, even though not significant, spindles in the MD 433 seem to start even earlier with respect to SOs as it is the case in the ANT. Again, in order 434 to project spindles to the neocortex that nest in neocortical up states in a timely manner, 435 a shifted coordination of SOs and spindles in the MD is necessary.

436 Taken together, our findings provide first evidence for the ANT as a major hub for 437 coordinating the cardinal NREM-sleep related oscillations. In the neocortex, both spindles 438 and SOs were led by ANT-related activity, while the interplay of local ANT SO-spindles 439 was tuned in a way that allows for neocortical spindles to nest within SO up states. 440 Importantly, this exact coordination is thought to be instrumental for the consolidation of 441 memories ${ }^{66,67}$.

442 Due to its projections to the medial prefrontal cortex and the hippocampus, the ANT has 443 already been implicated in facilitating memory functioning and spatial navigation during 444 wake, putatively by facilitating hippocampal-neocortical interactions ${ }^{25,27,68-70}$. Notably, the 445 same hippocampal-cortical dialogue is assumed to underly sleep-related memory 446 consolidation, facilitated by a precise coupling of hippocampal ripples, neocortical SOs 447 and thalamic spindles ${ }^{71}$. The standard model suggests that neocortical SOs are imposed 
448 on a rather passive thalamus, where they initiate spindles. Spindles in turn are thought to

449 synchronize hippocampal ripples and reactivated memory information ${ }^{19,67}$. Through this

450 coordination the reactivated memory information is suggested to arrive at the neocortex

451 during periods of high plasticity (i.e., during the presence of SO up states and spindles)

$452 \quad 72,73$.

453 Our data critically extend these theoretical considerations, by spotlighting the human

454 thalamus, and in specific the ANT, as a putative active agent in interfacing sleep-related

455 oscillations between brain regions. While our current data remain agnostic with regards

456 to hippocampal activity, it has been shown in a single patient study, that hippocampal

457 spindles are aligned with SOs in the ANT ${ }^{74}$. Hence, ANT related oscillations might not

458 only migrate to the neocortex but likewise to the hippocampus, where spindles are known

459 to govern ripples and associated memory reactivation ${ }^{66,75}$.Taken together, ANT related

460 activity might critically contribute to the triple coupling of sleep oscillations and thus, the

461 memory function of sleep. Future work will need to capture the association of ANT-

462 neocortical interactions with the behavioral expressions of memory consolidation, as well

463 as continue characterizing the functionally diverse human thalamus.

464

465 


\section{METHODS}

\section{Participants.}

469 Intracranial and scalp EEG were simultaneously recorded from patients with bilateral 470 depth electrodes implanted in the thalamus for deep brain stimulation (DBS) therapy of 471 pharmaco-resistant epilepsy. Data was recorded at the Epilepsy Center, Department of 472 Neurology, Ludwig-Maximilian Universität (Munich, Germany), while DBS leads were 473 externalized post-surgery, prior to their connection to the pulse generator located in a 474 subcutaneous infraclavicular or abdominal pouch ${ }^{76}$. The availability of direct iEEG 475 recordings from the human thalamus is highly limited due to (i) the rarity of patients being 476 treated with thalamic DBS, (ii) access to thalamic iEEG in these patients (post-surgery 477 externalization of DBS leads is performed only in a fraction of these patients), and (iii) the 478 limited amount of time for externalization (after surgery and before connecting the leads 479 to the pulse generator).

480 A total of 13 patients participated. The data of 5 patients were discarded due to excessive 481 epileptic activity. Out of the remaining 8 patients (mean age: $38.65 \pm 3.59 ; 5$ female), 6 482 patients contributed single full-night recordings, one patient contributed two consecutive 483 full-night recordings and one patient contributed three consecutive full-night recordings. 484 In sum, 11 full-night recordings entered the analyses of the present study. All patients 485 received anticonvulsive medication (see Supplementary Table 7 and 8 for each 486 participant's drug regimen at the time of recordings and their influence on sleep 487 architecture). Informed consent was obtained from all patients. The study was approved 488 by the ethics committee of the Medical Faculty of the Ludwig-Maximilian Universität.

Intracranial data acquisition and electrode localization

491 iEEG data was recorded from thalamic depth electrodes that each had four intracranial 492 electrode contacts (platinum-iridium contacts, $1.5 \mathrm{~mm}$ wide with $1.5 \mathrm{~mm}$ edge-to-edge 493 distance; Medtronic 3387). Data were recorded using XLTEK Neuroworks software 494 (Natus Medical, San Carlos, California, US) and an XLTEK EMU128FS amplifier, with 495 voltages referenced online to a $\mathrm{CPz}(250 \mathrm{~Hz}$ sampling rate in 7 patients; $200 \mathrm{~Hz}$ sampling 496 rate in 1 patient).

497 The ANT in both hemispheres are the clinically-relevant implantation target for DBS 498 therapy of epilepsy ${ }^{77,78}$. Due their small and high inter- and intraindividual variable size 499 and the transventricular implantation trajectory, a subset of the electrode contacts ends 500 up outside the ANT, frequently in the mediodorsal thalamus (see Fig. 1a). 
501 The locations of the electrode contacts were estimated using the Lead-DBS toolbox ${ }^{79}$.

502 First, the post-operative CT scan was co-registered to the pre-operative T1-weighted

$503 \mathrm{MRI}$, as implemented in the Advanced Normalisation Tools ${ }^{80}$. The scans were then

504 spatially normalized to MNI space, based on the pre-operative T1 image using the unified

505 segmentation approach as implemented in SPM12 ${ }^{81}$. Next, the trajectories of the DBS

506 electrodes and positions of the electrode contacts were reconstructed based on the

507 post-operative CT scan. The positions of the electrode contacts where then visually

508 confirmed using the DISTAL atlas ${ }^{82}$. Based on this localization, the $\mathrm{iEEG}$ recordings were

509 re-referenced to their immediate neighboring contact (bipolar montage). A bipolar pair of

510 contacts was considered to be in the ANT if both or at least one contact was localized

511 to the ANT, but no contact was localized in the MD. A bipolar pair of contacts was

512 considered to be in the MD if both or at least one contact was localized to the MD, but

513 no contact was localized in the ANT ${ }^{83}$.

514 This procedure yielded 21 thalamic contacts (7 patients) in the ANT, and 24 contacts (8

515 patients) in the MD (see Supplementary Fig. 2 for a comparison of left and right ANT /

516 MD contacts with regards to occurrence probabilities of SOs and spindles).

\section{Scalp EEG acquisition}

519 Scalp EEG was simultaneously recorded with iEEG via a common amplifier. Scalp EEG 520 electrodes were placed on the accessible scalp according to the international 10-20

521 system for electrode placement. The sampling rate of the scalp EEG was identical to the 522 sampling rate of the iEEG. Scalp EEG was recorded from 20 (2 patients), 22 (3 patients), 52324 (1 patient) or 36 electrodes (2 patients). Scalp EEG was online referenced to CPz.

524 Scalp EEG data was re-referenced offline against the mean of all available artifact-free 525 scalp EEG electrodes.

\section{$527 \quad$ Sleep staging}

528 All available scalp electrodes were low-pass filtered at $40 \mathrm{~Hz}$, down-sampled to $200 \mathrm{~Hz}$ 529 (if necessary). Sleep staging was carried out according to standard criteria ${ }^{84}$. For 530 subsequent analyses, sleep stages N2 and N3 were collapsed and referred to as non531 rapid eye movement (NREM) sleep.

\section{Data pre-processing}

534 iEEG and scalp EEG were manually inspected to discard noisy or artefact-contaminated 535 (e.g., excessive movements during NREM) channels (see below for detection of epileptic 
536 activity). All data were down-sampled to $200 \mathrm{~Hz}$, demeaned and de-trended. The

537 recordings were then filtered using a $150 \mathrm{~Hz}$ Butterworth low-pass filter and a two

538 Butterworth band-stop filters (to attenuate line noise; 48-52 Hz).

539

540 Interictal epileptic discharge (IED) detection: Epileptic activity in scalp and thalamic

541 recordings was semi-automatically detected. First, interictal epileptic discharges were

542 detected automatically by high- and low pass filtering the data below 20 and above 80

$543 \mathrm{~Hz}$ and z-scoring the data. Subsequently, data segments with amplitudes exceeding the

544 mean signal by 6 SDs for less than $100 \mathrm{~ms}^{85,86}$ were identified. Second, iEEG and scalp

545 EEG data was visually inspected for epileptic activity by two investigators, independently.

546 Data segments comprising epileptic events at any given channel were discarded (13.91

$547 \pm 2.41 \%$ of all sleep data). Four thalamic contacts (2 ANT contacts, 2 MD contacts) and

548 on average $7.5 \pm 0.92$ scalp electrodes were discarded.

\section{Event detection}

551 SOs and sleep spindles were identified for each patient, based on established detection

552 algorithms ${ }^{19,32}$.

553 SO detection: Data were filtered between 0.3-2 Hz (two-pass FIR bandpass filter, order

$554=$ three cycles of the low frequency cut-off). Only movement-free data (as determined

555 during sleep scoring) from NREM sleep stages 2 and 3 were considered. All zero-

556 crossings were determined in the filtered signal of each channel (iEEG and scalp EEG,

557 respectively), and event duration was determined for SO candidates (that is, down states

558 followed by up states) as time between two successive positive- to-negative zero-

559 crossings. Events that met the SO duration criteria (minimum of 0.8 and maximum of 2

$560 \mathrm{sec}$ ) and exceeded the mean amplitude of all detected events by 1.25 SD entered the

561 analysis. For subsequent time-locked analyses (ERPs, time-frequency representations,

562 etc.), 5 -sec-long segments centered on the down state ( $\pm 2.5 \mathrm{sec})$ were extracted from

563 the unfiltered raw signal.

564 Spindle detection: Data was filtered between 11-16 Hz (two-pass FIR bandpass filter,

565 order $=$ three cycles of the low frequency cut-off). Again, only artifact-free data from

566 NREM sleep stages 2 and 3 were used for event detection. The root mean square (RMS)

567 signal was calculated for the filtered signal at each channel using a moving average of

$568200 \mathrm{~ms}$, and spindles that exceeded the mean amplitude of all detected events by 1.5

569 SD entered the analysis. Whenever the signal exceeded this threshold for more than 0.5 570 sec but less than $3 \mathrm{sec}$ (duration criteria), a spindle event was detected. Epochs time- 
571 locked to the maximally negative spindle trough $(-2.5$ to $+2.5 \mathrm{sec})$ were extracted from

572 the unfiltered raw signal for subsequent time-locked analyses.

573 SO-spindle complexes: To isolate SO-spindle complexes in scalp and thalamic 574 recordings, we determined for all SOs whether a spindle was detected following the SO

575 (SO down state $+750 \mathrm{~ms}$ ). Again, SO-spindle events were extracted ( -2.5 to $+2.5 \mathrm{sec}$ 576 with regards to the SO down state) from the raw signal for subsequent time-locked 577 analyses.

578 Paired events: To identify paired SO events (scalp \& thalamus), we determined for all 579 scalp detected SOs whether corresponding SOs were detectable in thalamic recordings 580 within a time-window of $\pm 750 \mathrm{~ms}$. Data segments locked to the scalp detected down 581 states $( \pm 2.5 \mathrm{sec}$ ) were extracted. Likewise, paired sleep spindles were isolated by 582 determining for all scalp detected sleep spindles whether related spindles emerged $( \pm$ $583750 \mathrm{~ms}$ ) in thalamic recordings. Data segments locked to the scalp detected maximally 584 negative spindle peak $( \pm 2.5 \mathrm{sec})$ were extracted.

Electrode selection [scalp EEG]: As outlined above, all electrodes exhibiting significant epileptic activity were discarded to ensure that our results were not influenced by 588 epileptiform activity. This conservative procedure together with restricted access to the 589 scalp due to post-surgical wound care impeded us from using identical scalp EEG 590 electrodes across patients to delineate thalamo-cortical interactions during sleep (e.g., 591 electrode $\mathrm{Fz}$ for SO related and electrode Cz for sleep spindle related analyses ${ }^{18,19}$. 592 Instead, we pursued a data-driven and individualized approach. For SO-related analyses, 593 we selected for each participant the frontal scalp electrode that exhibited the highest 594 number of associated thalamic SOs within a time of $\pm 750 \mathrm{~ms}$. This procedure was 595 accomplished independently for data stemming from the ANT and the MD. The 596 respective scalp electrodes (one related to the ANT, one related to the MD) were used 597 for all subsequent SO-related analyses (see Supplementary Table 1 for on overview of 598 the selected scalp electrode; see Supplementary Fig. 3 for ANT SO occurrence 599 probabilities relative to scalp electrode Fz). Similarly, for sleep spindle related analyses, 600 we selected the frontal, central or parietal scalp electrode that exhibited the highest 601 number of associated thalamic spindles (ANT or MD, respectively; see Supplementary 602 Table 2 for on overview of the selected scalp electrodes; see Supplementary Fig. 4 for 603 spindle-spindle occurrence probabilities, were scalp spindles were exclusively derived 604 from frontal or parietal electrodes). The respective scalp electrodes (one related to the 
605 ANT, one related to the MD) were subsequently used for all sleep spindle-related

606 analyses. Data from scalp electrodes was used as a proxy of neocortical activity.

607

608 Event-free segments: For statistical comparisons, we extracted 5-sec-long intervals

609 during NREM sleep, which did not exhibit any SO, spindle or SO-spindle event, 610 respectively. In each participant and electrode, the number of control events 611 corresponded to the number of prior detected events of interest. Event-free events were 612 only drawn from time window starting 5 mins before and ending 5 mins after the 613 corresponding oscillatory event.

614

615 Analyses

616 Temporal relation between sleep oscillations: To assess the temporal relationship 617 between scalp- and thalamus-derived SO events, we created peri-event time histograms 618 (bin size $=50 \mathrm{~ms}$ ) where scalp detected SO down states served as seed (time $=0$ ), while 619 the targets (thalamic SO down states) are depicted relative to the seed. The resulting 620 histogram was normalized by the total number of detected scalp SOs (multiplied by 100).

621 Similarly, for sleep spindles, peri-event histograms (bin size $=50 \mathrm{~ms}$ ) of thalamic sleep 622 spindle peaks (maximally negative amplitude) in relation to scalp sleep spindles peaks 623 (maximally negative amplitude: $\pm 1.5 \mathrm{sec}$ ) were created. The resulting histogram was 624 normalized by the total number of detected scalp spindles (multiplied by 100). To 625 determine the interplay of SOs and sleep spindles (separately within scalp and thalamic 626 recording), a peri-event histogram of sleep spindles (onsets) around SO down states ( \pm $6271.5 \mathrm{sec}$ ) was created. The resulting histogram was normalized by the total number of 628 detected spindles (multiplied by 100). The same procedure was applied to spindle-free 629 control segments.

631 Phase relationship between sleep oscillations: To estimate the phase-relationship 632 between scalp and thalamic SOs, data epochs including paired events were filtered in 633 the SO range (0.3-2 Hz, two-pass Butterworth bandpass filter). As described above, 634 these segments included both scalp and thalamic SOs, and were time-locked to the 635 down states of scalp detected SOs. A Hilbert transform was applied to the data and the 636 instantaneous phase angle of the thalamic recording at the time of the scalp SO down 637 state was extracted. Each participant's preferred phase of SO-SO coupling was then 638 obtained by taking the circular mean of all individual events' preferred phases. 
639 For the analysis of SO-spindle coupling, we filtered the SO-spindle data (locked to spindle

640 onsets) in the SO range (0.3-2 Hz, two-pass Butterworth bandpass filter), applied a

641 Hilbert transform and extracted the instantaneous phase angle. Next, we isolated the SO

642 phase angle at the time of spindle onsets. Each participant's t preferred SO phase at

643 spindle onsets was obtained by taking the circular mean of all individual events' preferred

644 phases.

645

646 Spindle related across-channel power correlations: We further estimated the temporal

647 relationship of thalamic and scalp detected spindles using channel to channel correlations

648 of oscillatory power in the sleep spindle band. To this end, a Hanning window was applied

649 to paired sleep spindles segments (locked to scalp detected maximally negative

650 amplitude) and power values between 11 and $16 \mathrm{~Hz}$ were extracted for each time-bin.

651 Power values were z-scored per channel and averaged. Subsequently, power values

652 derived from thalamic channels were correlated with scalp related power values at each

653 time-point (Spearman correlation), resulting in a 2d correlation map per contact pair.

654 Finally, the correlation maps were normalized using the Fisher z-transform. The same

655 procedure was applied to spindle-free control segments. Off-diagonal correlations

656 indicate a time-lag between spindle power in scalp and thalamic channels.

658 Phase Slope Index: We assessed whether SOs drive activity in the sleep spindle range 659 or vice versa in NREM sleep data segments comprising SO-spindle complexes, 660 separately for thalamic and scalp recordings. The cross-frequency phase-slope index ${ }^{34}$, 661 was calculated for each contact between the signal and the signal filtered in the sleep 662 spindle range $(11-16 \mathrm{~Hz})$. After applying a Hanning window and extracting the complex 663 Fourier coefficients, all SO frequencies $<2 \mathrm{~Hz}$ were considered. In this context, positive 664 values indicate SOs driving sleep spindle activity, while negative values indicate sleep 665 spindles driving SOs. The obtained data distributions were tested against zero, using 666 paired samples t-tests.

667

668 Time frequency representations: Time-frequency analyses of SO, sleep spindle and SO669 spindle segments were performed using FieldTrip ${ }^{87}$. Frequency decomposition of the 670 data was achieved using Fourier analysis based on sliding time windows (moving forward 671 in $50 \mathrm{~ms}$ increments). The window length was set to five cycles of a given frequency 672 (frequency range: $1-30 \mathrm{~Hz}$ in $1 \mathrm{~Hz}$ steps). The windowed data segments were multiplied 673 with a Hanning taper before Fourier analysis [ -2.5 to $2.5 \mathrm{sec}$ ]. The longer time segments 
674 were chosen to allow for resolving low frequency activity within the time windows of

675 interest [ -1.5 to $1.5 \mathrm{sec}]$ and avoid edge artifacts. Resulting power values were z-scored

676 across time. The same procedure was applied to event-free control segments.

677

678 Statistics: Statistical analyses were performed at the individual electrodes / contacts level 679 (fixed-effects analysis), considering all electrodes / contacts that were eligible based on 680 our criteria (e.g., artefact free, etc.). Unless stated otherwise, we used cluster-based 681 permutation tests to correct for multiple comparisons as implemented in fieldtrip. A 682 dependent-samples t-test was used at the sample level and values were thresholded at $683 p=0.05$ (1000 randomizations). The sum of all t-values in cluster served as cluster 684 statistic and Monte Carlo simulations were used to calculate the cluster p-value (alpha = 685 0.05, two-tailed) under the permutation distribution. The input data were either 686 occurrence probabilities across time (e.g., Fig. 2a), time x frequency values (e.g., Fig. 2c 687 ) or time $x$ time correlation maps (e.g., Fig. 4b) which were tested against corresponding 688 data stemming from event-free events. In case of between area comparisons (e.g., Fig. $6893 \mathrm{~g}$ ) independent samples t-tests (cluster corrected) were employed to conform with the 690 varying number of observation units (i.e., contacts).

691 For circular statistics the phase distributions (within or across participants) were tested 692 against uniformity using the Rayleigh test (CircStat toolbox ${ }^{88}$ ). When directly comparing 693 phase distributions across recording sites, the circular Watson-Williams test was used $69488,89$. 


\section{References:}

702 1. Mölle, M., Bergmann, T. O., Marshall, L. \& Born, J. Fast and slow spindles during

703 the sleep slow oscillation: disparate coalescence and engagement in memory

$704 \quad$ processing. Sleep 34, 1411-21 (2011).

705 2. Piantoni, G. et al. Modulation of gamma and spindle-range power by slow

706 oscillations in scalp sleep EEG of children. Int. J. Psychophysiol. (2013). doi:10.1016/j.ijpsycho.2013.01.017

3. Klinzing, J. G., Niethard, N. \& Born, J. Mechanisms of systems memory consolidation during sleep. Nature Neuroscience (2019). doi:10.1038/s41593019-0467-3

4. Amzica, F. \& Steriade, M. The functional significance of K-complexes. Sleep Med. Rev. 6, 139-149 (2002).

5. Steriade, M., McCormick, D. A. \& Sejnowski, T. J. Thalamocortical oscillations in the sleeping and aroused brain. Science (80-. ). (1993). doi:10.1126/science.8235588

6. Timofeev, I., Grenier, F., Bazhenov, M., Sejnowski, T. J. \& Steriade, M. Origin of slow cortical oscillations in deafferented cortical slabs. Cereb. Cortex (2000). doi:10.1093/cercor/10.12.1185

7. Hughes, S. W., Cope, D. W., Blethyn, K. L. \& Crunelli, V. Cellular Mechanisms of the Slow (. Neuron (2002).

8. David, F. et al. Essential thalamic contribution to slow waves of natural sleep. J. Neurosci. (2013). doi:10.1523/JNEUROSCI.3169-13.2013

9. Gent, T. C., Bandarabadi, M., Herrera, C. G. \& Adamantidis, A. R. Thalamic dual control of sleep and wakefulness. Nat. Neurosci. (2018). doi:10.1038/s41593018-0164-7

10. Crunelli, V. \& Hughes, S. W. The slow $(<1 \mathrm{~Hz})$ rhythm of non-REM sleep: a dialogue between three cardinal oscillators. Nat. Neurosci. 13, 9 (2010).

11. Crunelli, V., David, F., Lorincz, M. L. \& Hughes, S. W. The thalamocortical network as a single slow wave-generating unit. Curr. Opin. Neurobiol. 31, 72-80 (2015).

12. Neske, G. T. The Slow Oscillation in Cortical and Thalamic Networks: Mechanisms and Functions. Front. Neural Circuits 9, (2016).

13. Steriade, M. Grouping of brain rhythms in corticothalamic systems. Neuroscience 137, 1087-1106 (2006).

14. Helfrich, R. F., Mander, B. A., Jagust, W. J., Knight, R. T. \& Walker, M. P. Old Brains Come Uncoupled in Sleep: Slow Wave-Spindle Synchrony, Brain Atrophy, and Forgetting. Neuron 97, 221-230.e4 (2018).

15. Mak-McCully, R. A. et al. Coordination of cortical and thalamic activity during non-REM sleep in humans. Nat. Commun. 8, 15499 (2017).

16. Mölle, M., Marshall, L., Gais, S. \& Born, J. Grouping of spindle activity during slow oscillations in human non-rapid eye movement sleep. J. Neurosci. 22 , 10941-7 (2002).

17. Sirota, A., Csicsvari, J., Buhl, D. \& Buzsáki, G. Communication between neocortex and hippocampus during sleep in rodents. Proc. Natl. Acad. Sci. U. S. A. 100, 2065-2069 (2003).

18. Clemens, fia et al. Temporal coupling of parahippocampal ripples, sleep spindles and slow oscillations in humans. doi:10.1093/brain/awm146

19. Staresina, B. P. et al. Hierarchical nesting of slow oscillations, spindles and ripples in the human hippocampus during sleep. Nat. Neurosci. 18, 1679-1686 (2015).

20. Rasch, B. \& Born, J. About sleep's role in memory. Physiol. Rev. 93, 681-766 (2013). 
21. Schreiner, T. \& Staudigl, T. Electrophysiological signatures of memory reactivation in humans. Philosophical Transactions of the Royal Society B: Biological Sciences 375, (2020).

22. Girardeau, G. \& Lopes-dos-Santos, V. Brain neural patterns and the memory function of sleep. Science (80-. ). 374, 560-564 (2021).

23. Pergola, G. et al. The Regulatory Role of the Human Mediodorsal Thalamus. Trends Cogn. Sci. 22, 1011-1025 (2018).

24. Aggleton, J. P., Pralus, A., Nelson, A. J. D. \& Hornberger, M. Thalamic pathology and memory loss in early Alzheimer's disease: moving the focus from the medial temporal lobe to Papez circuit. Brain 139, 1877-1890 (2016).

25. Aggleton, J. P. \& Brown, M. W. Episodic memory, amnesia, and the hippocampal-anterior thalamic axis. Behav. Brain Sci. 22, 425-444 (1999).

26. Fernandez, L. M. J. \& Lüthi, A. Sleep Spindles: Mechanisms and Functions. Physiological reviews 100, 805-868 (2020).

27. Viejo, G. \& Peyrache, A. Precise coupling of the thalamic head-direction system to hippocampal ripples. Nat. Commun. $202011111,1-14$ (2020).

28. Varela, C. \& Wilson, M. A. Mpfc spindle cycles organize sparse thalamic activation and recently active ca1 cells during non-rem sleep. Elife (2020). doi:10.7554/eLife.48881

29. Yang, M., Logothetis, N. K. \& Eschenko, O. Occurrence of Hippocampal Ripples is Associated with Activity Suppression in the Mediodorsal Thalamic Nucleus. J. Neurosci. (2019). doi:10.1523/jneurosci.2107-18.2018

30. Benjamini, Y. \& Hochberg, Y. Controlling the False Discovery Rate: A Practical and Powerful Approach to Multiple Testing. Source J. R. Stat. Soc. Ser. B 57, 289-300 (1995).

31. Hahn, M. A., Heib, D., Schabus, M., Hoedlmoser, K. \& Helfrich, R. F. Slow oscillation-spindle coupling predicts enhanced memory formation from childhood to adolescence. Elife 9, 1-21 (2020).

32. Schreiner, T., Petzka, M., Staudigl, T. \& Staresina, B. P. Endogenous memory reactivation during sleep in humans is clocked by slow oscillation-spindle complexes. Nat. Commun. (2021). doi:10.1038/s41467-021-23520-2

33. Nolte, G. et al. Robustly estimating the flow direction of information in complex physical systems. Phys. Rev. Lett. 100, 234101 (2008).

34. Jiang, H., Bahramisharif, A., van Gerven, M. A. J. \& Jensen, O. Measuring directionality between neuronal oscillations of different frequencies. Neuroimage 118, 359-367 (2015).

35. Marzetti, L., Del Gratta, C. \& Nolte, G. Understanding brain connectivity from EEG data by identifying systems composed of interacting sources. Neuroimage 42, 87-98 (2008).

36. Mölle, M. \& Born, J. Slow oscillations orchestrating fast oscillations and memory consolidation. 193, 93-110 (2011).

37. Adamantidis, A. R., Gutierrez Herrera, C. \& Gent, T. C. Oscillating circuitries in the sleeping brain. Nat. Rev. Neurosci. 20, 746-762 (2019).

38. Blethyn, K. L., Hughes, S. W., Tóth, T. I., Cope, D. W. \& Crunelli, V. Neuronal Basis of the Slow $(\& 1 t ; 1 \mathrm{~Hz})$ Oscillation in Neurons of the Nucleus Reticularis Thalami In Vitro. J. Neurosci. 26, 2474-2486 (2006).

39. Contreras, D. \& Steriade, M. Cellular basis of EEG slow rhythms: a study of dynamic corticothalamic relationships. J. Neurosci. 15, 604-22 (1995).

40. Slézia, A., Hangya, B., Ulbert, I. \& Acsády, L. Phase Advancement and NucleusSpecific Timing of Thalamocortical Activity during Slow Cortical Oscillation. J. Neurosci. 31, 607-617 (2011).

41. Poulet, J. F. A., Fernandez, L. M. J., Crochet, S. \& Petersen, C. C. H. Thalamic 
control of cortical states. Nat. Neurosci. 2012153 15, 370-372 (2012).

42. Hay, Y. A. et al. Thalamus mediates neocortical Down state transition via GABA B-receptor-targeting interneurons. Neuron 109, 2682-2690.e5 (2021).

43. Pare, D., Steriade, M., Deschenes, M. \& Oakson, G. Physiological characteristics of anterior thalamic nuclei, a group devoid of inputs from reticular thalamic nucleus. https://doi.org/10.1152/jn. 1987.57.6.1669 57, 1669-1685 (1987).

44. Gonzalo-Ruiz, A. \& Lieberman, A. R. Topographic organization of projections from the thalamic reticular nucleus to the anterior thalamic nuclei in the rat. Brain Res. Bull. 37, 17-35 (1995).

45. Halassa, M. M. \& Sherman, S. M. Thalamocortical Circuit Motifs: A General Framework. Neuron 103, 762-770 (2019).

46. Jankowski, M. M. et al. The anterior thalamus provides a subcortical circuit supporting memory and spatial navigation. Front. Syst. Neurosci. 7, (2013).

47. Grodd, W., Kumar, V. J., Schüz, A., Lindig, T. \& Scheffler, K. The anterior and medial thalamic nuclei and the human limbic system: tracing the structural connectivity using diffusion-weighted imaging. Sci. Reports 2020101 10, 1-25 (2020).

48. Van Groen, T. \& Wyss, J. M. Projections from the anterodorsal and anteroveniral nucleus of the thalamus to the limbic cortex in the rat. J. Comp. Neurol. 358 , 584-604 (1995).

49. Hicks, R. R. \& Huerta, M. F. Differential thalamic connectivity of rostral and caudal parts of cortical area Fr2 in rats. Brain Res. 568, 325-329 (1991).

50. Massimini, M., Huber, R., Ferrarelli, F., Hill, S. \& Tononi, G. The sleep slow oscillation as a traveling wave. J. Neurosci. 24, 6862-70 (2004).

51. Nir, Y. et al. Regional Slow Waves and Spindles in Human Sleep. Neuron 70, 153-169 (2011).

52. Riedner, B. A., Hulse, B. K., Murphy, M. J., Ferrarelli, F. \& Tononi, G. Temporal dynamics of cortical sources underlying spontaneous and peripherally evoked slow waves. Prog. Brain Res. 193, 201-218 (2011).

53. De Gennaro, L. \& Ferrara, M. Sleep spindles: an overview. Sleep Med. Rev. 7, 423-440 (2003).

54. Steriade, M. \& Deschenes, M. The thalamus as a neuronal oscillator. Brain Res. 320, 1-63 (1984).

55. Halassa, M. M. \& Acsády, L. Thalamic Inhibition: Diverse Sources, Diverse Scales. Trends Neurosci. 39, 680-693 (2016).

56. Gentet, L. J. \& Ulrich, D. Strong, reliable and precise synaptic connections between thalamic relay cells and neurones of the nucleus reticularis in juvenile rats. J. Physiol. 546, 801-811 (2003).

57. Zikopoulos, B. \& Barbas, H. Parallel driving and modulatory pathways link the prefrontal cortex and thalamus. PLoS One 2, (2007).

58. Velayos, J. L., Oliva, M. \& Alfageme, F. Afferent projections to the mediodorsal and anterior thalamic nuclei in the cat. Anatomical-clinical correlations. Brain Pathol. 8, 549-552 (1998).

59. Latchoumane, C.-F. V., Ngo, H.-V. V., Born, J. \& Shin, H.-S. Thalamic Spindles Promote Memory Formation during Sleep through Triple Phase-Locking of Cortical, Thalamic, and Hippocampal Rhythms. Neuron 1-12 (2017). doi:10.1016/j.neuron.2017.06.025

60. Buchmann, A. et al. Reduced mediodorsal thalamic volume and prefrontal cortical spindle activity in schizophrenia. Neuroimage 102 Pt 2, 540-547 (2014).

61. Ferrarelli, F. \& Tononi, G. Reduced sleep spindle activity point to a TRN-MD thalamus-PFC circuit dysfunction in schizophrenia. Schizophr. Res. 180, 36-43 (2017). 
62. Bastuji, H., Lamouroux, P., Villalba, M., Magnin, M. \& Garcia-Larrea, L. Local sleep spindles in the human thalamus. J. Physiol. 598, 2109-2124 (2020).

63. Mölle, M., Marshall, L., Gais, S. \& Born, J. Grouping of spindle activity during slow oscillations in human non-rapid eye movement sleep. J. Neurosci. 22 , 10941-10947 (2002).

64. Mikutta, C. et al. Phase-amplitude coupling of sleep slow oscillatory and spindle activity correlates with overnight memory consolidation. J. Sleep Res. 28, (2019).

65. Zhang, J., Yetton, B., Whitehurst, L. N., Naji, M. \& Mednick, S. C. The effect of zolpidem on memory consolidation over a night of sleep. Sleep (2020). doi:10.1093/sleep/zsaa084

66. Kim, J., Gulati, T. \& Ganguly, K. Competing Roles of Slow Oscillations and Delta Waves in Memory Consolidation versus Forgetting. Cell 179, 514-526.e13 (2019).

67. Maingret, N., Girardeau, G., Todorova, R., Goutierre, M. \& Zugaro, M. Hippocampo-cortical coupling mediates memory consolidation during sleep. Nat. Neurosci. 19, 959-964 (2016).

68. Taube, J. S. Head direction cells recorded in the anterior thalamic nuclei of freely moving rats. J. Neurosci. 15, 70-86 (1995).

69. Peyrache, A., Lacroix, M. M., Petersen, P. C. \& Buzsáki, G. Internally organized mechanisms of the head direction sense. Nat. Neurosci. 18, 569-575 (2015).

70. Leszczyński, M. \& Staudigl, T. Memory-guided attention in the anterior thalamus. Neurosci. Biobehav. Rev. 66, 163-165 (2016).

71. Diekelmann, S. \& Born, J. The memory function of sleep. Nat. Rev. Neurosci. 11, 114-126 (2010).

72. Niethard, N., Ngo, H. V. V., Ehrlich, I. \& Born, J. Cortical circuit activity underlying sleep slow oscillations and spindles. Proc. Natl. Acad. Sci. U. S. A. (2018). doi:10.1073/pnas.1805517115

73. Seibt, J. et al. Cortical dendritic activity correlates with spindle-rich oscillations during sleep in rodents. Nat. Commun. 8, (2017).

74. Sarasso, S. et al. Hippocampal sleep spindles preceding neocortical sleep onset in humans. Neuroimage 86, 425-432 (2014).

75. Ngo, H.-V. V, Fell, J. \& Staresina, B. P. Sleep spindles mediate hippocampalneocortical coupling during sharp-wave ripples. bioRxiv 712463 (2019). doi:10.1101/712463

76. Kaufmann, E., Bötzel, K., Vollmar, C., Mehrkens, J. H. \& Noachtar, S. What have we learned from 8 years of deep brain stimulation of the anterior thalamic nucleus? Experiences and insights of a single center. J. Neurosurg. 135, 619628 (2020).

77. Fisher, R. et al. Electrical stimulation of the anterior nucleus of thalamus for treatment of refractory epilepsy. Epilepsia (2010). doi:10.1111/j.15281167.2010.02536.x

78. Kaufmann, E. et al. European Expert Opinion on ANT-DBS therapy for patients with drug-resistant epilepsy (a Delphi consensus). Seizure (2020). doi:10.1016/j.seizure.2020.08.015

79. Horn, A. \& Kühn, A. A. Lead-DBS: a toolbox for deep brain stimulation electrode localizations and visualizations. Neuroimage 107, 127-135 (2015).

80. Avants, B. B., Epstein, C. L., Grossman, M. \& Gee, J. C. Symmetric diffeomorphic image registration with cross-correlation: evaluating automated labeling of elderly and neurodegenerative brain. Med. Image Anal. 12, 26-41 (2008).

81. Ashburner, J. \& Friston, K. J. Unified segmentation. Neuroimage 26, 839-851 (2005). 
82. Ewert, S. et al. Toward defining deep brain stimulation targets in MNI space: A subcortical atlas based on multimodal MRI, histology and structural connectivity. Neuroimage 170, 271-282 (2018).

83. Deutschová, B. et al. Thalamic oscillatory activity may predict response to deep brain stimulation of the anterior nuclei of the thalamus. Epilepsia (2021). doi:10.1111/epi.16883

84. Iber, C., Ancoli-Israel, S., Chesson, A. \& Quan, S. The AASM Manual for the Scoring of Sleep and Associated Events: Rules, Terminology, and Technical Specification. American Academy of Sleep Medicine (2007).

85. Andrillon, T. et al. Sleep spindles in humans: insights from intracranial EEG and unit recordings. J. Neurosci. 31, 17821-17834 (2011).

86. Helfrich, R. F. et al. Bidirectional prefrontal-hippocampal dynamics organize information transfer during sleep in humans. Nat. Commun. (2019). doi:10.1038/s41467-019-11444-x

87. Oostenveld, R., Fries, P., Maris, E. \& Schoffelen, J.-M. FieldTrip: Open source software for advanced analysis of MEG, EEG, and invasive electrophysiological data. Comput. Intell. Neurosci. 2011, 156869 (2011).

88. Berens, P. CircStat: A MATLAB toolbox for circular statistics. J. Stat. Softw. 31, 1-21 (2009).

89. VanRullen, R. How to Evaluate Phase Differences between Trial Groups in Ongoing Electrophysiological Signals. Front. Neurosci. 10, (2016).

\section{Acknowledgments}

This work was supported by the European Research Council (https://erc.europa.eu/, Starting Grant 802681 awarded to TSt). We are indebted to all patients who volunteered to participate in this study. We thank the staff and physicians of the at the Epilepsy Center, Department of Neurology, Ludwig Maximilians University, Munich for assistance.

\section{Author Contributions}

Conceptualization: TS, TSt; Resources: EK, NS, JHM; Formal Analysis: TS, TSt; Funding acquisition: TSt; Writing - original draft: TS, TSt; Writing- review \& editing: TS, TSs, EK, SN

\section{Corresponding author}

Correspondence to Tobias Staudigl

\section{Competing interests}

The authors declare no competing interests. 
947 Supplementary Information for:

948

949 The human thalamus orchestrates neocortical oscillations during

950 NREM sleep.

951

952 Thomas Schreiner ${ }^{1}$, Elisabeth Kaufmann², Soheyl Noachtar ${ }^{2}$, Jan-Hinnerk 953 Mehrkens $^{3}$ \& Tobias Staudigl ${ }^{1}$

954

9551 Department of Psychology, Ludwig-Maximilians-Universität München, Munich,

956 Germany

9572 Epilepsy Center, Department of Neurology, Ludwig-Maximilians-Universität München, 958 Munich, Germany

959

9603 Department of Neurosurgery, Ludwig-Maximilians-Universität München, Munich, 961 Germany

962

963

964

965

966

967

968

969

970

971

972

973

974

975

976

977

978

979

980 


\section{Supplementary tables}

Supplementary Table 1 | Sleep architecture: Data are means \pm s.e.m. N1, N2: NREM sleep stages N1 \& N2, SWS: slow-wave sleep, REM: rapid eye movement sleep, WASO: wake after sleep onset. TST: total sleep time (in minutes).

\begin{tabular}{c|cccccc} 
& $\mathrm{N} 1$ & $\mathrm{~N} 2$ & SWS & REM & WASO & TST [min] \\
\hline Sleep stage [\%] & $6.1 \pm 1.7$ & $47.4 \pm 4.2$ & $16.5 \pm 3.5$ & $12.7 \pm 2.2$ & $15.9 \pm 3.5$ & $529.4 \pm 30.1$
\end{tabular}

Supplementary Table 2 | scalp electrodes: overview of scalp electrode that were selected for the

990

\begin{tabular}{l|cccc} 
& ANT-SO & ANT-spindle & MD-SO & MD-spindle \\
\hline P1 & Fz & Fp1 & Fz & P4 \\
P2 & F4 & F4 & FP2 & Pz \\
P3 & F8 & F4 & F4 & F4 \\
P4 & F110 & Pz & FT10 & C3 \\
P5 & F4 & Pz & F3 & P3 \\
P6 & Fz & C3 & Fz & Pz \\
P7 & F110 & C4 & Fz & Pz \\
P8 & - & - & F7 & Cz
\end{tabular}

Supplementary Table 3 | Sleep Oscillations ANT: Data are means \pm s.e.m. Number of detected events and percentage of paired events in NREM sleep at ANT contacts and scalp electrodes.

\begin{tabular}{r|cccc} 
& ANT & Scalp & $t$ & $P$ \\
\hline SO number & $1573.8 \pm 129.9$ & $1513.5 \pm 130.1$ & 1.11 & 0.29 \\
SO-coupling [\%] & $33.74 \pm 3.02$ & $31.11 \pm 3.25$ & 2.07 & 0.06 \\
spindle number & $2203.8 \pm 213.5$ & $1903.3 \pm 211.5$ & 3.19 & 0.008 \\
spindle-coupling [\%] & $43.8 \pm 4.2$ & $37.3 \pm 4.1$ & 3.43 & 0.005 \\
SO-spindle number & $431 \pm 52.1$ & $304.1 \pm 69.8$ & 3.18 & 0.008
\end{tabular}
and percentage of paired events in NREM sleep at MD contacts and scalp electrodes.

\begin{tabular}{r|cccc} 
& MD & Scalp & $t$ & $P$ \\
\hline SO number & $1408.5 \pm 126.2$ & $1476.9 \pm 129.1$ & 1.06 & 0.31 \\
SO-coupling [\%] & $30.45 \pm 2.31$ & $29.51 \pm 3.15$ & 1.37 & 0.35 \\
Spindle number & $2172.8 \pm 238.2$ & $1796.5 \pm 229.8$ & 3.35 & 0.005 \\
spindle-coupling [\%] & $45.1 \pm 5.5$ & $30.2 \pm 4.6$ & 2.18 & 0.046 \\
SO-spindle number & $488 \pm 53.5$ & $338.5 \pm 79.7$ & 1.78 & 0.095
\end{tabular}


bioRxiv preprint doi: https://doi.org/10.1101/2021.12.11.471766; this version posted December 12, 2021. The copyright holder for this preprint (which was not certified by peer review) is the author/funder, who has granted bioRxiv a license to display the preprint in perpetuity. It is made available under aCC-BY-NC-ND 4.0 International license.

Supplementary Table 5 | SO features: Data are means \pm s.e.m. Duration and relative occurrence during $\mathrm{N} 2$ and N3 sleep for scalp (ANT and MD analyses related), ANT and MD derived SOs.

\begin{tabular}{r|ccc} 
SO & Duration [sec] & Rel. N2 [\%] & Rel. N3 [\%] \\
\hline scalp ANT & $1.31 \pm 0.01$ & $46.56 \pm 3.79$ & $53.43 \pm 3.79$ \\
scalp MD & $1.31 \pm 0.02$ & $47.41 \pm 4.38$ & $52.58 \pm 4.38$ \\
ANT & $1.30 \pm 0.01$ & $46.05 \pm 3.68$ & $53.94 \pm 3.68$ \\
MD & $1.31 \pm 0.02$ & $47.18 \pm 4.93$ & $52.81 \pm 4.93$
\end{tabular}

1007

1008

1009

1010 1011

1012

1013

1014 Supplementary Table 7 | drug regimen at the time of recordings.

\begin{tabular}{l|c} 
& Anticonvulsant \\
\hline P1 & Levetiracetam, Phenytoin, Lamotrigine \\
P2 & Levetiracetam, Lamotrigine \\
P3 & Lacosamide, Oxcarbazepine, Topiramate \\
P4 & Levetiracetam, Lamotrigine, Oxcarbazepin, Zonisamide \\
P5 & Levetiracetam, Lamotrigine \\
P6 & Lamotrigine, Lacosamide, Zonisamide \\
P7 & Oxcarbazepin, Topiramate \\
P8 & Topiramate
\end{tabular}

1015

1016

1017

1018

1019

1020

1021

1022

1023

1024

1025

Supplementary Table 6 | spindle features: Data are means \pm s.e.m. Peak frequency, duration and relative occurrence during N2 and N3 sleep for scalp (ANT and MD analyses related), ANT and MD derived spindles

Supplementary Table 8 | Documented effects drugs on sleep architecture.

\begin{tabular}{l|c} 
Drug & Effect on sleep \\
\hline Lacosamide $^{1}$ & none \\
Lamotrigine $^{2}$ & REM $\uparrow$, N2个, SWS $\downarrow$ \\
Levetiracetam $^{3}$ & N2个, REM $\downarrow$ \\
Oxcarbazepine & unknown \\
Phenytoin $^{4}$ & SWS $\downarrow$, REM $\downarrow$ \\
Topiramate $^{5}$ & none \\
Zonisamide $^{6}$ & none
\end{tabular}

1) Hudson J.D., Guptil J.T., Bynes W., et al. (2015). Assessment of the effects of lacosamide on sleep parameters in healthy subjects. Seizure; 25: 155-159 2) Placidi F, Marciani MG, Diomedi M, et al (2000). Effects of lamotrigine on nocturnal sleep, daytime somnolence and cognitive functions in focal epilepsy. Acta Neurol Scand;102:81-86. 3) Cicolin A, Magliola U, Giordano A, et al. (2006). Effects of levetiracetam on nocturnal sleep and daytime vigilance in healthy volunteers. Epilepsia; 47:82-85. 4) Benjamin Legros \& Carl W Bazil (2003). Effects of antiepileptic drugs on sleep architecture: a pilot study. Sleep Med; 4(1):51-5. 5) Bonanni E, Galli R, Maestri M, et al. (2004). Daytime sleepiness in epilepsy patients receiving topiramate monotherapy. Epilepsia; 45:333-337. 6) Romigi A, Izzi F, Placidi $F$, et al (2013). Effects of zonisamide as add-on therapy on sleep-wake cycle in focal epilepsy: a polysomnographic study. Epilepsy Behav; 26:170-174.

\begin{tabular}{r|cccc} 
spindles & Peak-Freq [Hz] & Duration [ms] & Rel. N2 [\%] & Rel. N3 [\%] \\
\hline scalp ANT & $13.02 \pm 0.15$ & $0.81 \pm 0.03$ & $73.71 \pm 3.81$ & $26.29 \pm 3.81$ \\
scalp mD & $13.07 \pm 0.13$ & $0.77 \pm 0.05$ & $70.68 \pm 5.14$ & $29.32 \pm 5.14$ \\
ANT & $13.17 \pm 0.09$ & $0.79 \pm 0.04$ & $73.51 \pm 3.76$ & $26.49 \pm 3.76$ \\
MD & $13.03 \pm 0.11$ & $0.78 \pm 0.03$ & $74.02 \pm 3.49$ & $25.97 \pm 3.49$
\end{tabular}




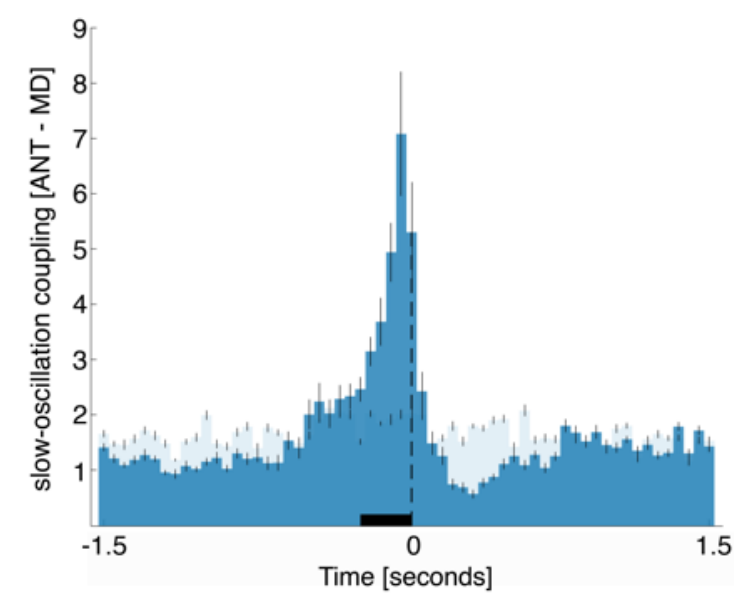

Supplementary Fig. 1 | SO coupling ANT-MD: Occurrence probabilities of ANT SO down state peaks relative to MD SO down state peaks (time $=0$; dashed line), indicating that ANT SOs precede on average MD SOs, when directly compared (i.e., without taking neocortical coordination into account, as in Fig. 2
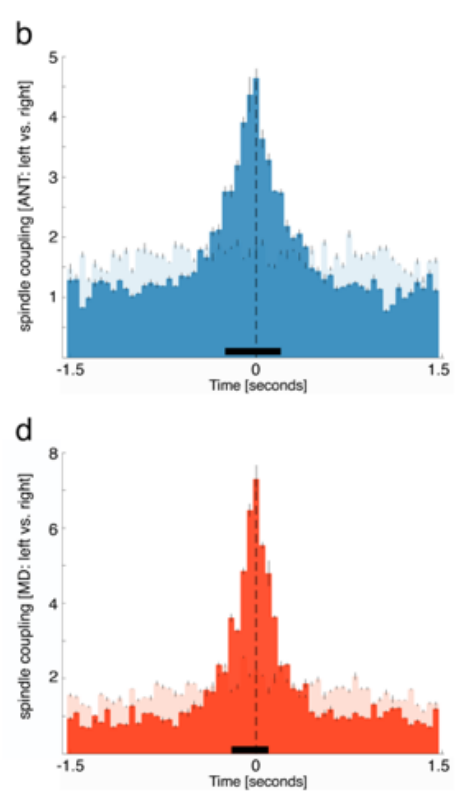

Supplementary Fig. 2 | Lateralization of coupling: (a) Occurrence probabilities of left ANT SO down state peaks relative to right ANT SO down state peaks, indicated that SOs in the left and right ANT emerged synchronously (significant positive cluster from -0.1 to $0.1 \mathrm{sec}$; $<<0.0001$; time of peak $=0 \mathrm{sec}$ ). (b) Occurrence probabilities for sleep spindles derived from the left ANT, relative to right ANT detected spindles, indicated that SOs in the left and right ANT emerged synchronously (significant positive cluster from -0.25 to $0.25 \mathrm{sec} ; \mathrm{p}<0.0001$; time of peak = $0 \mathrm{sec}$ ). (c) Occurrence probabilities of left MD SO down state peaks relative to right MD SO down state peaks, (significant positive cluster from -0.15 to $0.05 \mathrm{sec}$; $p<0.0001$; time of peak $=0 \mathrm{sec}$ ). (d) Occurrence probabilities for sleep spindles derived from the left $M D$, relative to right $M D$ detected spindles (significant positive cluster from -0.2 to $0.1 \mathrm{sec} ; \mathrm{p}<0.0001$; time of peak $=0 \mathrm{sec}$ ). 
1047

1048
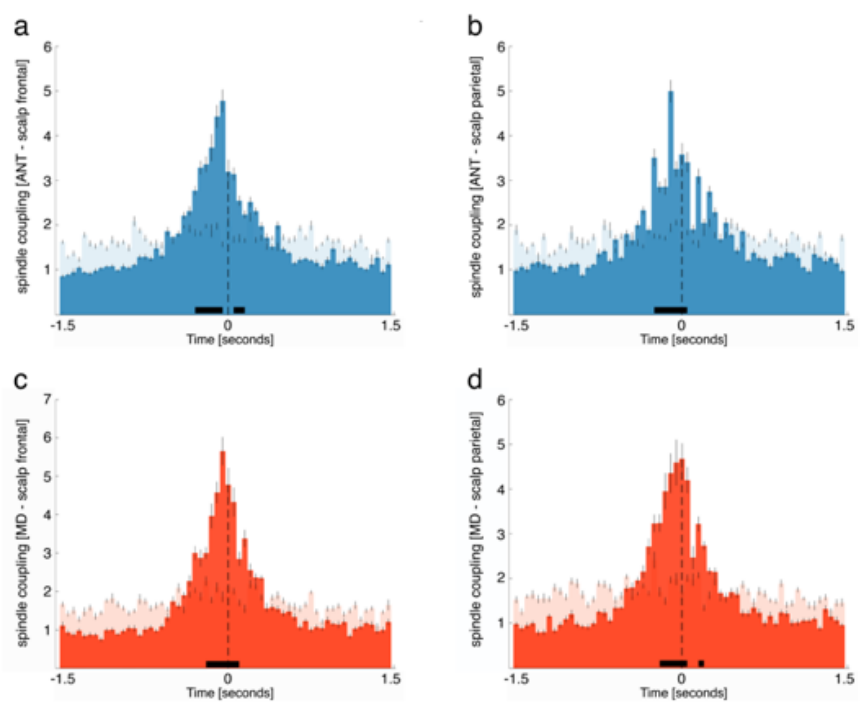

Supplementary Fig. 4 | Thalamic spindles coupled to frontal and parietal scalp spindles: $(a+b)$ Occurrence probabilities of ANT spindle peaks relative to frontal (a) and parietal (b) neocortical spindle peaks (maximal negative amplitude, time $=0$ ), indicating that ANT spindles precede neocortical spindles detected from both frontal and parietal scalp electrodes (frontal: first significant positive cluster from 0.3 to $-0.05 \mathrm{sec}, \mathrm{p}<0.001$; second cluster from 0.05 to $0.15 \mathrm{sec}, \mathrm{p}=0.005$; time of peak: $-0.05 \mathrm{sec}$; parietal: significant positive cluster from -0.25 to $0.05 s, p<0.001$ ). ( $c+d)$ Occurrence probabilities of MD spindle peaks relative to frontal (c) and parietal (d) neocortical spindle peaks (maximal negative amplitude, time $=0$ ), indicating that MD spindles precede neocortical spindles detected from both frontal and parietal scalp electrodes (frontal: significant positive cluster from -0.2 to $0.1 \mathrm{sec}, p<0.001$; time of peak: $-0.05 \mathrm{sec}$; parietal: first significant positive cluster from -0.2 to $0.05 \mathrm{sec}, p<0.001$; second significant positive cluster from 0.15 to $0.2 \mathrm{sec}, \mathrm{p}=0.01$; time of peak $=0$ ). 